Aus der dermatologischen Klinik von Prof. Tommasoli

in Palermo.

\title{
Ueber Embolie und Metastase in der Haut.
}

\author{
Von \\ Dr. L. Philippson, \\ Assistent. \\ (Hierzu Taf. I-VI.)
}

Nach den Lehren der allgemeinen Pathologie spielt bei der Ausbreitung der Krankheiten im Organismus der Transport pathogener Stoffe auf dem Blutwege eine grosse Rolle. Daher macht man auch in der medicinischen Klinik und in der pathologischen Anatomie den ausgiebigsten Gebrauch von den Gesetzen der Embolie und der Metastase in den Fällen, wo es sich darum handelt, Organerkrankungen zu erklären, für deren Entstehung man nicht die nothwendigen Bedingungen in ihnen selbst vorfindet. Alsdann sucht man nämlich nach einem anderswo Jocalisirten primären Krankheitsherde, von dem aus die Krankheitsursache in das Blut, durch welches sie Verbreitung. im Körper findet, gelangen kann.

Anders in der Dermatologie. Hier zieht man den erwähnten Entstehungsmodus für krankhafte Hautveränderungen verhältnismässig selten in Betracht und scheint er nur auf gewisse bakterielle Krankheiten und auf Granulome anwendbar $\mathrm{zu}$ sein.

Nun gelangen aber doch auch in der dermatologischen Klinik viele Hautkrankheiten zur Beobachtung, die mit inneren Krankheiten in Beziehung stehen, wie erklärt man alsdann dieses Abhängigkeitsverhältniss? Der Regel nach sind es die Nerren, welche als Vermittler zwischen der innern Krankheit und der Haut herangezogen werden. Man denke nur an die 
pathogenetische Erklärung, welche man von den Hautreränderungen bei den acuten Exanthemen, bei acuten Infectionskrankheiten, bei den Arzneiexanthemen, bei den Erythrodermieen, bei allen Arten von Erythemen, bei Pemphigus, bei Dermatitis herpetiformis, bei vielen anderen bullösen Dermatitiden, bei Prurigo und vielen anderen pruriginösen Erkrankungen u. a. m. gibt und man wird mir Recht geben, wenn ich behaupte, dass in der Dermatologie die Lehre von der Ausbreitung der Krankheiten auf dem Nervenwege ihrer Bedeutung nach die Stelle, welche die Lehre von der Embolie und Metastase in der allgemeinen Pathologie einnimmt, vertritt.

Dieser Umstand würde noch deutlicher hervortreten, wenn nicht auch auf einem anderen Gebiete in der Dermatologie so häufig auf Nerven recurrirt würde, nämlich auf dem Gebiete der Aetiologie. Ohne in Uebertreibung zu verfallen, kann man wohl sagen, dass es keine Hautkrankheit mit unbekannter Aetiologie gibt, bei welcher nicht schon die Nerven als ursächliches Moment herangezogen wurden oder noch heute herangezogen werden. Dies mag wohl ein Grund dafür gewesen sein, dass, wie gesagt, bei der Pathogenese die Nerven eine so grosse Rolle spielen, denn als Ursachen von Krankheiten können sie ihrer Natur nach auch eine Fernwirkung ausüben und man braucht daher, um eine solche zu erkläron, dann nicht mehr nach einem anderen anatomischen Substrat zu suchen.

Sollten nun aber wirklich in der Pathologie der Hautkrankheiten andere Gesetze gelten als in der übrigen Medicin?

Durch eine Reihe von Untersuchungen bin ich allmälig zu der Ueberzeugung gelangt, dass dies nicht der Fall ist: auch in der Hautpathologie sind embolische und metastatische Vorgänge sehr gewöhnliche Vorkommnisse. Wenn dies bisher nicht erkannt worden ist, so lag es eben an der neuropathologischen Richtung, welche in der Dermatologie herrschend ist. Ganz besonders ist es die Lehre von den Angioneurosen gewesen, welche der richtigen Erkenntniss hindernd im Wege stand, und kann ich wohl sagen, dass es die eingehende Beschäftigung gerade mit diesen Processen gewesen ist, die mich auf die richtige Spur gebracht und mir das Gebiet der Embolie and Metastase in der Hautpathologie erschlossen hat. 
Die allgemeine Pathologie dieser Vorgänge nun in den Hauptzügen zu entwerfen, ist die Aufgabe, welche ich mir in dieser Publication gestellt habe.

Was die Untersuchungen selbst betrifft, aus denen ich die allgemeinen Sätze abstrahire, so sind sie zum grössten Theile schon veröffentlicht worden und darf ich mich daher wohl auf dieselben beziehen. Um aber meine Auseinandersetzungen nicht durch zu häufiges Citiren unterbrechen zu müssen, ziehe ich es vor, meine diesbezüglichen Arbeiten gesondert zusammenzustellen und aus ihnen kurz diejenigen Thatsachen hervorzuheben, die für unser Thema wichtig sind.

Da nun die Erytheme einen Haupttheil meiner Untersuchungen ausmachen, ich aber dieselben in ganz anderer Weise auffasse, als es heute in der Dermatologie gelehrt wird, so erachte ich es für nothwendig, bereits von vornherein die Angioneurosenlehre einer Kritik zu unterziehen, um mich nicht später immer mit derselben auseinandersetzen zu müssen.

Vorliegende Arbeit zerfallt demnach in drei Theile:

1. Kritik der Angioneurosenlehre.

2. Zusammenstellung meiner Publicationen, die zu unserer Frage Beziehung haben.

3. Hauptsätze zur Lehre von der Embolie und Metastase in der Haut.

\section{Kritik der Angioneurosenlehre.}

Als ich dem Ursprunge der Angioneurosenlehre in der Literatur nachforschte, fühlte ich mich enttäuscht von der schwachen thatsächlichen Grundlage, welche dieselbe besitzt. Der Begriff der Angioneurose ist nämlich nicht aus klinischen Beobachtungen hergeleitet worden, sondern, wie aus der ersten Publication von Eulenburg, dem Schöpfer dieses Namens, erhellt (Ueber cutane Angioneurosen, Berl. klin. Woch. 1867) wurde er nur als Pendant zur Trophoneurose gebildet. Gerade so wie die trophischen Nerven erkranken, könnten, so sagte er, auch die vasomotorischen Nerven erkranken und dafür würde dann Angioneurose das passende Wort sein. Um nun von dieser theoretischen Speculation eine Anwendung auf die Klinik zu machen, vermuthete der Autor, dass z. B. in dem Herpes Zoster 
eine Angioneurose vorläge. Von den heutzutage hierher gerechneten Krankheiten aber spricht er gar nicht, sondern erwähnt nur flüchtig Urticaria.

Dies ist der eigentliche Ausgangspunkt der Angioneurosenlehre gewesen, die dann später auf dermatologischem Gebiete eine so weitgebende Anwendung fand, dass eine grosse Gruppe von Krankheiten unter sie gefallen ist, an die bei Aufstellung des Begriffes ganz und gar nicht gedacht wurde.

Sind nun aber im Laufe der Zeit Beweise für die Richtigkeit dieser Auffassung beigebracht worden? So viel ich sehe, nicht. Denn daraus, dass mit dieser Lehre gewisse Eigenschaften der betreffenden Krankheiten erklärt werden können, darf man doch nicht umgekehrt auf die Richtigkeit derselben schliessen. Dieser Mangel an thatsächlichen Beweisen ist es nun, der zur Kritik herausfordert und zur Neubearbeitung des ganzen Gebietes hindrängt.

Zwar ist die Angioneurosenlehre schon häufiger Gegenstand wissenschaftlichen Disputes gewesen, aber selbst diejenigen, welche derselben nicht die Bedeutung zumessen wollten, die ihr von Köbner und Lew in zugesprochen worden war, fahren fort in anderer Weise von ihr ausgiebigen Gebrauch zu machen, so dass auch bei ihnen auf dem Gebiete der Erytheme die Gefässnerven eine sehr wesentliche Rolle spielen.

Von der Richtigkeit dieser Behauptung wird man sich leicht überzeugen könuen, wenn man die Ansichten verschiedener Autoren über diesen Gegenstand mit einander vergleicht.

Für Köbner und Lew in besteht bekanntlich das Wesen der Erytheme in einer Störung des vasomotorischen Centrums. Auf Grund dieser gemeinsamen Ursache werden von ihnen die verschiedensten Krankbeiten als Angioneurosen vereinigt, sobald sie nur auf der Haut erythematöse Veränderungen bewirken.

Wenn nun auch gegen diese Auffassung Ka posi, Besnier und Doyon Einspruch erheben, so geschieht es doch nur, weil sie principiell bei der Classification von Krankbeiten ausser der Ursache auch anderer Momente Rechnung getragen wissen wollen. Nach ihnen soll man die Krankheiten nach ihrem Gesammtverlauf classificiren und nicht nach einem einzigen Factor. In Betreff der eigentlichen Hauptirage bei den Erythemen aber 
stimmen sie vollständig mit den ersten Autoren überein, auch für sie sind es angioneurotische Störangen.

Ueber die Art nun, wie dieselben zu Stande kommen, lassen sich Besnier und Doyon sehr ausführlich aus, indem sie auch die verschiedenen Ursachen, welche zu Erythemen Anlass geben, in Betracht ziehen. Sie kommen aber dabei zu dem Schlusse, dass für die Erytheme die Art der Ursachen ganz von nebensächlicher Bedeutung ist, da dieselben überhaupt nur als Nervenreize auf das vasomotorische Centrum zur Geltung kommen. Sie erkennen nun zwar an, dass bei Erythemen auch localer Gefässreflex vorkommen kann, aber sie betonen doch ausdrücklich, dass für das Auftreten von Erythemen die Anwesenheit des Krankheitsstoffes in der Haut nicht von nöthen sei; ja dass selbst der Befund von Mikroorganismen in den erythematösen Läsionen nicht gegen den allgemeinen Modus ihres Entstehens, nämlich auf reflectorischem Wege, sprechen würde. Und damit ist dann der Angioneurosenlehre wieder eine grosse Bedeutung zugeschrieben.

Wir sehen demnach bereits aus diesen wenigen Citaten, dass unter den Klinikern zwar sehr grosse Differenzen in Bezug auf den nosologischen Werth der Angioneurosenlehre bestehen, dass aber ihre Bedeutung für die Pathogenese der erythematösen Hautveränderungen unumwunden anerkannt wird.

Dieser Umstand könnte nun von nebensäcblicher Bedeutung zu sein scheinen bei Krankheiten. die nicht die Haut allein betreffen, sondern vielmehr Allgemeinerkrankungen mit Hautsymptomen vorstellen. Wenn man aber bedenkt, dass die sichersten Kenntnisse, welche wir von ihnen besitzen, zugleich diejenigen, an welchen wir den ganzen Krankheitsprocess erkennen, es sind dies gerade die Hautmanifestationen, so ist die Frage ihrer Pathogenese von grosser Wichtigkeit.

Diese eben gemachte Trennung zwischen den cutanen Veränderungen der Erytheme und dem ihnen zu Grunde liegenden Krankheitsprocesse im Innern führt uns zu einem Punkte, der hier ebenfalls berührt werden muss. Es will mir nämlich scheinen, als ob die ganze Erythemdiscussion auch deshalb an Unklarheit leidet, weil man gerade diese Trennung nicht gemacht hat. Denn gewöhnlich schliesst man hier so, dass man sagt, das 
Hauterythem ist angioneurotischer Natur und in Folge dessen ist die ganze Krankheit eine Angioneurose. Während doch die erste Hälfte des Satzes richtig sein könnte, wenn auch die innere Krankheit anderer Natur, z. B. infectiöser Natur wäre. Und ein anderer Fehler, der dem eben erörterten nahe steht, ist folgender. Nachdem man einmal angenommen hatte, dass in gewissen Fällen Erytheme angioneurotischer Natur wären, übertrug man diese Auffassung auch auf alle diesen gleichen oder ähnlichen Hautveränderungen, selbst da, wo der innere Krankheitsprocess seiner Aetiologie und Pathogenese nach sehr gut bekannt war, wie bei Septicämie, bei acuten und chronischen Infectionskrankheiten, ohne auch nur die Möglichkeit in Betracht zu ziehen, dass auch für die Haut dieselbe Aetiologie und Pathogenese massgebend sein könnte.

Dass man überhaupt so weit gehen konnte, erklärt sich nur daraus, dass man eben in den erythematösen Hautveränderungen etwas grundsätzlich Verschiedenes von anderen ähnlichen Veränderungen sah, nämlich von den gewöhnlichen entzündlichen. Aus diesem Grunde konnte man dann die Erytheme auch nicht auf die bekannten Ursachen der inneren Krankheiten zurückführen, sondern musste als Vermittler zwischen beiden die Nerven einschieben.

Und dieselbe Tendenz, die dazu führte, in den Hautsymptomen der Erytheme etwas ganz Besonderes zu sehen, tritt auch noch in anderer Weise hervor. Besnier und Doyon sehen nämlich als Eigenthümlichkeit der Erytheme den Umstand an, dass die individuelle Disposition für ihr Entstehen wichtiger ist, als die materielle Ursache, die ihnen zu Grunde liegt. Sie sagen damit, dass nicht den Ursachen als solchen die Fähigkeit innewohnt in jedem Organismus Erythem hervorzurufen, sondern dass Erytheme nur bei denjenigen Personen auftreten, die eine besondere Disposition dafür haben und dass die Art der Ursachen von ganz nebensächlicher Bedeutung ist. Sie berühren damit eine Frage, die überhaupt bej allen Krankheiten in Betracht gezogen wird, besonders aber bei denjenigen, deren Ursachen und Pathogenese so unvollständig bekannt sind, dass wir nicht im Stande sind, die Wirkung im voraus zu bestimmen.

Es sind also nicht die Erytheme allein, bei denen man 
auf die Disposition zurückgreifen muss, um die Krankheit zu erklären, sondern wir kennen in der Dermatologie auch andere Processe, bei denen ganz genau dieselben Verhältnisse vorliegen, ich meine die artificiellen Hautentzündungen. Auch bei ihnen besteht eine so grosse Incongruenz zwischen Ursache und Wirkung, dass wir hiebei die Annahme einer Idiosynkrasie nicht umgehen können.

Also auch mit Rïcksicht auf diesen Punkt kann ich keine principiellen Differenzen zwischen Erythemen und anderen ähnlichen Dermatosen erkennen, sondern finde ich sogar in dieser Beziehung eine vollständige Uebereinstimmung zwischen den Erythemen und den oberflächlichen Hautentzündungen.

Nachdem wir somit die Hauptfragen, die bei den Erythemen in Betracht kommen, haben Revue passiren lassen und nachdem wir darauf hingewiesen haben, dass die Lehre von der Angioneurose sehr schwach begründet ist, bleibt noch ein Gegenstand zu erörtern übrig, nämlich die pathologische Anatomie der Erytheme.

Zwar ist diese niemals herangezogen worden um die herrschende Lehre zu beweisen oder zu unterstützen, aber wir können doch trotzdem verlangen, dass die histologischen Befunde wenigstens nicht in Widerspruch mit der klinischen Auffassung stehen.

Das aber scheint mir gerade aus den histologischen Beschreibungen hervorzugehen, die von den Anhängern der Angioneurosen selbst gegeben werden. Die Befunde, welche bei den Efflorescenzen der Erytheme gemacht wurden, waren nämlich eine kleinzellige Infiltration, auch rothe Blutkörperchen im Gewebe und Oedem des Bindegewebes, Befunde, die doch den einfachen bekannten Entzïndungsprocessen zukommen. Trotz alledem folgert aber Kaposi daraus, dass die Erythempapel nur eine stärker entwickelte Urticariaquaddel ist, was so viel sagen will, dass sie, wie diese, angioneurotischer Natur ist. Auch Lewin sieht in diesen Veränderungen eine reine Angioneurose. Selbst Unna, der zwar Entzündung diagnosticirt, findet. Eigenthümlichkeiten in ihr, die ihn dazu führen, dieselbe neurotische Entzündung zu nennen. Nur Campana erklärt die Befunde einfach für die Wirkung eines exsudativen Processes. 
Sehen wir also von der Deutung, welche die Autoren den von ihnen gemachten histologischen Thatsachen geben, ab und halten wir uns nur an diese selbst, so sind dieselben morphologisch den entzündlichen gleich.

Kennen wir nun aus der Physiologie Thatsachen, wonach die vasomotorischen Nerven im Stande sind derartige Veränderungen hervorzurufen? Oder sind derartige Befunde schon bei angioneurotischen Erkrankungen, die sonst in der Pathologie bekannt sind, gemacht worden? So viel ich weiss, ist das nicht der Fall. Die Wirkung dieser Nerven beschränkt sich stets nur darauf, Congestion mit einer leicht vermehrten Transsudation hervorzurufen, aber eine kleinzellige Infiltration fehlt dabei vollständig.

Wenn demnach trotz der leicht zu constatirenden entzündlichen Natur der Erythemläsionen der Haut an dem angioneurotischen Charakter derselben festgehalten wird, so geht daraus deutlich hervor, wie feste Wurzeln die Angioneurosendoctrin in der Dermatologie geschlagen hat.

Würde ich nun aus der Histologie den Schluss ziehen, dass der erythematöse Process überhaupt nichts anderes als ein entzündlicher sei, so müsste ich mich natürlich àuf Einwürfe gefasst machen, die darauf abzielen, immer wieder die herrschende Lehre $\mathrm{zu}$ vertheidigen.

Man könnte mir z. B. einwenden, dass doch wenigstens die Blutkreislaufstörungen der Erytheme auf vasomotorischen Einfluss zurückgeführt werden müssten.

Aber auch dies zugegeben, was würde daraus folgen? Kommt der etwa nicht auch bei der Entzündung zur Geltung? Denn, wenn wir auch heute für die wesentlichste Bedingurg für das Entstehen der Entzündung die Gefässwandalteration im Sinne von Samuel und Cohnheim ansehen, so wissen wir doch andererseits auch aus den Experimenten über Entzündung, welch' grossen Einfluss die sympathischen Nerven auf das $\mathrm{Zu}-$ standekommen der congestiven Hyperämie hat, die neben der Gefässveränderung vorhanden sein muss, damit sich Entzündung entwickle. Aber trotzdem ist die Entzündung keine Angioneurose, denn die Sympathicuslähmung allein ruft zwar Congestion hervor, aber keine Entzündung. 
Und dieselbe Argumentation scheint mir auch auf die Erytheme anwendbar zu sein. Auch hier hängt die Hyperämie von den Vasomotoren ab, aber eine rasomotorische Störung allein bringt es nicht $\mathrm{zu}$ dem schon klinisch so hervortretenden Oedem und bewirkt keine Auswanderung weisser Blutkörperchen. Also auch hier bedürfen wir eines neuen Factors und den könnten wir beispielsweise ebenfalls in einer Gefässwandalteration, ähnlich der bei Entzündung vorkommenden, suchen.

Man könnte mir noch entgegenhalten, dass selbst zugegeben, dass bei gewissen Erythemen wirklich ein Entzündungsoder ein ihr nahestehender Process vorläge, es doch Erythemformen gibt, bei denen die anatomischen Veränderungen nicht den entzündlichen entsprechen, weil eben Emigration fehlt und die deshalb allen Erfordernissen angioneurotischer Störungen genügen, wie z. B. Urticaria.

Hierauf müsste ich jetzt eigentlich die Antwort schuldig bleiben, da ich mich bei dieser Kritik noch nicht auf eigene Untersuchungen beziehe, sondern nur die schwachen Seiten und die inneren Widersprüche der heute giltigen Lehre nachweisen wollte. Wie ich aber trotzdem hinzufügen will, habe ich mir auf Grund experimenteller Untersuchungen auch über Urticaria eine andere Meinung gebildet und erkenne ihr eine Pathogenese zu, die derjenigen der Erytheme sehr nahe steht.

Also auch diese Stiitze scheint mir der Angioneurosenlehre abhanden zu kommen, so dass sie gerade auf dem Gebiete, auf welchem sie bisher hauptsächlich Anwendung gefunden hat, hinfällig wird.

Wie aus dieser Kritik horvorgeht, kann ich der herrschenden Auffassung von den Erythemen ganz und gar nicht beistimmen. Meine abweichenden Ansichten habe ich im Laufe der Discussion schon angedeutet; da aber für das Verständniss der in dieser Arbeit zu behandelnden Fragen von Wichtigkeit ist, dass im Geiste des Lesers nicht der mindeste Zweifel über meine Stellung zn den Erythemen herrsche, so scheint es mir angebracht, noch einmal kurz, meinen Standpunkt zu präcisiren. Die Theorie der Angioneurosen, wie sie heute in der Dermatologie gelehrt wird, weise ich vollständig als unbegrïndet und unzureichend zurück. 
In Folge dessen erkenne ich auch nicht die Aufstellung einer besonderen Krankheitsgruppe, als Angioneurosen, an.

Und dies umsoweniger, als für mich die in den Lehrbüchern als Krankheiten beschriebenen Erytheme iiberhaupt nicht die Bedeutung von Krankheiten haben, sondern weiter nichts als im Verlaufe von inneren Erkrankungen vorkommende Hautveränderungen, als klinische Symptomencomplexe, vorstellen. Denn krankhafte Vorgänge werden wir nur dann mit Nutzen als Krankheiten in der Klinik auffassen, wenn wir aus ihrer Diagnose Schlüsse über den weiteren Verlauf, über den Ausgang, über die Ursacheu oder über ihre Bedingungen im allgemeinen ziehen können. Dies ist aber bei den Erythemen nicht der Fall. Erstens ist die Diagnose der Erytheme nur eine rein morphologische, wenn wir von Erythema multiforme oder papulatum oder nodosum oder von Urticaria sprechen, und zweitens sagen wir mit dieser Diagnose gar nichts über die einzelnen Factoren aus, die den ganzen krankhaften Vorgang zusammensetzen. Alle diese unterschiedenen Formen von Erythemen haben also nur den Sinn von klinischen Befunden in der Haut, die man unter sehr verschiedenen Krankheitsbedingungen machen kann.

Ich ziebe folglich die Erytheme einzig und allein als klinisch-anatomische Hautveränderungen in Betracht. Als solche aber und nur als solche habe ich sie einer eigenen Untersuchung für werth erachtet und mich mit ihnen in einer Reihe von Arbeiten besehäftigt.

\section{Zusammenstellung meiner sich auf Embolie und Metastase der Haut beziehenden Publicationen.}

Auf den ersten Blick mag es scheinen, als ob hier ganz heterogene Gegenstände zusammengestellt worden wären, aber in Wirklichkeit stehen sie doch alle miteinander in Verbindung. Sie betreffen nämlich die erythematösen Hautveränderungen, welche bei idiopathischen Erythemen und bei Infectionskrankbeiten vorkommen und auf deren Bedeutung für die uns hier beschäftigende Frage ich schon hingewiesen habe. Oder sie beziehen sich direct auf embolische Processe und liefern daher Beispiele für derartige Vorgänge. Auch Gefässveränderungen, die ich im Verlaufe anderer Untersuchungen bei verschiedenen 
Hautkrankheiten gefunden habe, gehören hierher, weil dadurch die Metastasenbildung bewiesen wird.

Man wird daher erkennen können, dass alle diese Publicationen, wenn sie auch zu verschiedenen Zeiten entstanden und in verschiedener Absicht gemacht worden sind, trotzdem darauf abzielen, Thatsachen auf einem Gebiet zu sammeln, das bisher so gut wie brach gelegen hat.

1. Die Histologie deracutentstehenden hyperämischen Flecke der Lepra tuberosa. (Virchow's Archiv, 1893, pag. 229.)

Bei einem leprösen Knaben hatte ich Gelegenheit, zu verschiedenen Malen acute, fieberhafte Ausbrüche der bekannten hyperämischen Flecke in der Haut zu beobachten und konnte ich daher dieselben in möglichst frühem Stadium zur histologischen Untersuchung bekommen. Durch den constanten Befund von Leprabacillen im Lumen der Capillaren wurde bewiesen, dass es sich hier um bacilläre Embolie in die Hautgefässe handelte. Die Flecken hatten also die Bedeutung von kleinen Entzündungsherden, die sich um die Embolie bildeten und die acute Eruption als Ganzes entsprach demnach einer Blutgefässmetastase des leprösen Processes in der Haut.

2. Ueber den anatomischen Bau einiger Granulationsgeschw ülste in der Haut. (Centralblatt f. Allgem. Path. u. Path. Anat. 1893, pag. 289.)

Versuch, die Frage zu beantworten, ob in der histologischen Structur von Hautläsionen der Infectionsmodus zum Ausdrucls kommt. Zu diesem Zwecke vergleiche ich die histologisehen Veränderungen, welche man bei nachweislich auf dem Blutwege entstandenen Krankheitsherden findet, nit den durch directe Infection von aussen hervorgerufenen, u. zw. bei Syphilis die Sclerose mit Roseola und Papeln, bei Lepra die acut entstehenden erythematösen Flecke mit gewissen oberflächlichen Knoten und bei Tuberculose verschiedene Arten von Lupus miteinander.

Ich komme zu folgenden Schlüssen: Bei Infection der Haut von aussen entstehen Granulome von oberfächlichem Sitz, die scharf von der Umgebung abgesetzt, mehr oder weniger kugelförmig sind und die erst später die tieferen Cutisschichten befallen. Ihre Wachsthumsrichtung ist eine radiäre; sie sind concentrisch gebaut.

Bei Infection der Haut a f dem Blutwege erstrecken sich die Neubildungen schon im Beginne ihrer Entwicklung durch alle Schichten der Cutis, da sie sich um die Blutgefässverzweigungen bilden, welche ihnen ihre Form vorschreiben. Sie sind dendritisch gebaut.

3. Allgemeine Diagnostik der Hautkrankheiten. (Bearbeitet in Gemeinschaft mit Dr. Török 1895.)

Aus diesem Buche interessirt uns hier das Capitel über Entzündung und Angioneurose. Gestützt auf die klinische Beobachtung der Entzündungsprocesse in der Haut kommen wir zu dem Schlusse, dass der clas- 
sische Symptomencomplex der Entzündung noch nicht die einfachste Form der Gefässreizung ist, sondern dass es in der Haut noch eine einfachere gibt, die wir reactive Reizerscheinungen nennen.

Diese bestehen klinisch einzig und allein aus congestiver Hyperämie mit oder ohne Oedem, wozu histologisch noch Emigration und der Fibringehalt des Exspdates kommen.

Bei der typischen Entzündung treten hierzu noch schwerere Gewebsveränderungen, wie Degenerationen, Eiterung, Nekrose etc.

Die erythematösen Hautveränderungen sind nun nichts anderes als reactive Reizerscheinungen der Gefässe und können demnach nicht als Angioneurosen den entzündlicben Krankheiten gegenübergestellt werden.

4. Contributo allo studio dell' Eritema nodoso. (Giorn. ital. mal. ven. e mal. della pelle 1895.)

Aus der histologischen Untersuchung von Hautläsionen in 3 Fällen von Erythema nodosum ergab sich, dass es sich hiebei um echte Entzündung handelt, bei der sich ausser der kleinzelligen Infiltration ein starkes, sero-fibrinöses Exsudat oder gar hämorrhagisches Exsudat vorfindet. Die angioneurotische Lebre des Erythems wird daber zurückgewiesen.

Gerade die Erytheme, u. zw, diejenigen, welche auf der Haut nicht in diffuser Form, sondern als isolirte, nmschriebene Herde auftreten, hatten für die mich interessirende Frage eine grosse Bedeutung, iusofern als ich gerade in ihnen den Typus der embolis chen Hautentzündung vermuthete. Diese Vermuthung war zwar in offenem Widerspruch mit der giltigen Lebre der Angioneurose, aber eine unbefangene Untersuchung: erwies ihre Berechtigung.

Nach den obigen ersten Untersuchungen habe ich dann noch später andere Formen von Erythemen und auch von Purpura untersucht und stets den entzündlichen Charakter derselben bestätigen können.

Aus diesen Resultaten konnte ich dann weiter entnehmen, dass die hämatogene Fntzündung in der Haut unter scheinbar sehr verschiedener Form auftritt und dass ausser der Localisation an der Oberfläche der Haut, auch eine primäre in dem Unterhautzellgewebe vorkommt. Die letztere Localisation der Entzündung hat noch ein besonderes Interesse, da sie sich auch, wie wir sehen werden, bei andersartigen Krankheitsprocessen wiederholt, denen aber dieselbe Genese wie dieser Entzündung zu Grunde liegt.

5. Sopra la Tromboflebite tubercolare entanea. (Giorn. ital. mal. ven. e della pelle 1898.)

Es handelt sich hier um einen Fall von sogenannten scrophulösen Gummen bei einem Individuum, welches auch an Drüseneiterungen am Halse und in den Achselhöblen litt. Während des längeren Aufenthaltes des Kranken im Hospital hatte ich Gelegenheit, die Entwicklung dieser Hautkrankheit genau zu verfolgen und erkannte so, dass dieselbe einen werthvollen Beitrag zu dem mich beschäftigenden Argumente lieferte. 
Es war mir schon auffällig bei der klinischen Beobachtung, dass es sich nicht um die gewöhnlichen Gummen handelte, die weiter nichts sind, um mich der in der Chirurgie üblichen Bezeichmung zu bedienen, als kalte Abscesse, die sich unter der Hant bilden, weil darunter liegende tuberculöse Organe verkäst sind, wie Gelenke, Knochen, Drüsen. In meinem Falle bestanden die Geschwüre gerade an den Beinen, wo an den unterliegenden Organen keine Tuberculose aufzufinden war. Ueber ibren Ursprung konnte ich aber erst ins Klare lsommen, als ich einen acuten Ausbruch von kleinen entzündlichen Herden an den Händen und Beinen beobachtete. Diese hatten meistens ibren Sitz in den tieferen Cutisschichten oder in der Subcutis. Die histologische Untersuchung ergab eine a cu te $P$ hlebitis, hervorgernfen durch Tuberkelbacillen. Die Weiterentwicklung der einzelnen Herde zeigte nun, dass an die Entzündung sich Nekrose, Eiterung anschloss und so die vorher sclon gesehenen Geschwüre entstanden.

Dieser Fall ist insofern lehrreich, als er ein neues Beispiel dafür abgibt von der Art, wie sich die Blutgefässmetastase in der Haut darstellt. Statt an den Arterien und den Capillaren finden wir die Hauptveränderungen an den Venen, in denen sich das Virus localisirt, Thrombose und Entzündung hervorruft und die somit den Ausgangspunkt des Krankheitsherdes bestimmen. Ausserdem abar lernen wir aus diesem Falle, dass unter dem klinischen Bilde von Erythemläsionen auch derartige schwerere Veränderungen, wie die Phlebitis verlaụfen können.

Ich habe bisher nur diesen einzigen Fall beobachtet, es scheint aber, dass ähnliche nicht so überaus selten sind, denn gewisse unter verschiedenen Namen publicirte Krankheitsfälle, die heute von Darier mit zu den $\mathrm{T}$ u berculides gerechnet werden, gleichen in ihren klinischen Ligenschaften dem meinigen sehr. Auch in diesen Fällen begannen die Hautveränderungen mit gerötheten, tief sitzenden Knötchen, in deren Centrum sich ein kleiner Abscess bildete, nach dessen Eröffnung dann ein sehr langsam heilendes Geschwür übrig blieb. Zugleich wird bei der Beschreibung dieser Fälle auf den Zusammenhang der Affection mit Tuberculose hingewiesen, da bei den betreffenden Kranken Lymphdrüsenoder Knochen- oder Lungenerkrankungen specifischer Natur vorlagen. Die bisher veröffentlichten histologischen Untersuchungen derartiger Fälle laben nun allerdings nicht die tuberculösen Eigenschaften der Hautveränderungen erkennen lassen und berichten auch nicht über den meinigen ähnliche Befunde, aber es scheint mir, dass bisher an recht unzureichendem Material gearbeitet wurde, so dass gerade die wichtigsten Thatsachen übersehen "werden konnten. Wenn man möglichst frische und bis in die Subcutis ausgeschnittene Läsionen zur Untersuchung genommen hätte, so glaube ich, würde man auch schon in den publicirten Fällen dieselben Veränderungen, wie ich sie angetroffen habe, gesehen haben. Aber auch heute, wo mein Fall allein steht, scheint er mir ein nicht zu unterschätzender Beitrag zu der Frage, die ich hier behanale.

Von diesem Falle stammen die Präparate, die in den Figuren 3 und 4 wiedergegeben sind. 
6. Delle Gomme sifilitiche esulla lorodipendenzada alterazioni vasali. (Giorn. Ital. mal, ven. e mal. della pelle 1898.)

Aus dieser Untersuchung interessirt uns hier der Befund an den Gefässen; ich konnte nämlich feststellen, dass der Ausgangspunkt des subcutanen syphilitischen Gummas zu suchen ist in einer Endophlebitis proliferans, mit welcher der Process beginnt und welche die Ausbreitung des Granuloms bestimmt.

Dass auch diese F'orm syphilitischer Hautaffection zu unserer Aufgabe Beziehung hat, wird man verstehen, wenn man berücksichtigt, dass jch danach suche, festzustellen, in welcher Form die Metastasenbildung in der Haut auftritt. Und da ich nun bereits früher darauf hin die primären und secundären Syphilodermen untersucht hatte, so blieben mir noch die tertiären übrig.

Um aber derartige Untersuchungen zu machen, bedarf es eines besonderen Naterials, nämlich möglichst frischer Krankheitsherde und diese sind im tertiären Stadium schwerer zu erhalten, als im secundären. Andererseits lagen in der Literatur nur zwei diesbezügliche Beschreibungen vor, eine von Cornil und eine andere von Marfau und Toupet, die sich wirklich auf den Beginn von Gummen beziehen, während alle anderen nur die späteren Rückbildungsvorgänge enthalten. Ich habe daher aus eigener Erfahrung die allerersten Veränderungen der Gummen kennen lernen wollen und habe dazu durch Zufall Gelegenheit gebabt, indem eine Patientin, die Jahre lang in der Klinik sich aufhielt, bäufiger an Gummen litt, die sie allmälig lernte, schon durch das Gefühl in dem Fettgewebe zu erkennen, wenn sie auch kaum die Grösse von Erbsen hatten.

Um nun in diesen Gummen die Gefässveränderung, deren Ausdehnung und Verhältniss zum Granulom genau festzustellen, ist es nöthig, den ganzen Krankheitsherd in Serienschnitten zu untersuchen.

Es ist dann leicht zu erkennen, dass das Gumma, d. h. das Granulom sich um eine Vene entwickelt, die durch Endothelwucherung vollständig verschlossen ist. Daraus ist zu schliessen, dass der Beginn der Läsion in einer Venenaffection zu suchen ist, die vom syphilitischen Virus abhängig ist oder mit anderen Worten: der hämatogene Ursprung auch dieses Syphiloderms ist histologisch erkennbar.

Man bemerke, dass es hier die Venen sind, die vorzugsweise befallen werden und nicht die Arterien, wie von den oben erwähnten Autoren behauptet wird. Ich habe zwar auch Endarteritis in Gummen gesehen, die ja in inneren Organen die gewöhnliche Form syphilitischer Gefässerkrankung zu sein scheint, aber in der Haut sind die Venenveränderungen nicht allein viel häutiger, sondern, wie aus meiner Untersuchung hervorgeht, auch die am frühesten auftretenden und um die sich später das Granulomgewebe entwickelt.

Um zu diesen sicheren Behauptungen zu kommen, habe ich mich, wie ich nicht vergessen will hinzuzufügen, nicht darauf beschränkt, Gummen von einer Kranken allein und von einer Körperregion allein, nämlich von den Beinen untersucht, sondern schon in dieser Mittheilung berich- 
tete ich über die Histologie von zwei anderen Gummen, die ich von zwei anderen Kranken und zwar von den Armen genommen Latte. Und da ich nach Veröffentlichung dieser Arbeit noch Gelegenheit hatte, zwei sehr junge Gummen, eines von der ersten Kranken und ein anderes von einem neuen Kranken, und auch dieses Mal von den Armen zu erhalten, so verfehlte ich nicht, von neuem eine Untersuchung vorzunehmen, die mir dasselbe Resultat ergab.

Die Figuren 5 und 6 beziehen sich auf diese beiden Fälle.

Dieser constante Befund von Gefässveränderungen bei Gummen forderte dazu auf, auch bei anderen Syphilodermen danach zu suchen. Von denjenigen der Frühperiode ist es bekannt, dass die entzündlichen Veränderungen nicht allein um die oberflächlichen, sondern auch um die tieferen Gefässe der Haut auftreten, eine Thatsache, die ich als Merkmal ihres hämatogenen Ursprungs angesehen habe. (Vergl. Nr. 2 dieser Zusammenstellung.)

Aber bei vielen anderen Syphilodermen ist ein derartiger Bau nicht zu erkennen, woraus hervorgeht, dass sie entweder eine andere Entstehungsart haben oder die ursprüngliche typische Anlage durch andere Factoren unkenntlich gemacht wird. Trotzdem ist es mir gelungen, auch bei nekrotischen Formen der Secundärperiode primäre Gefässveränderungen nachzuweisen, wodurch wiederum die MögTichkeit bewiesen wird, auch histologisch die embolische Natur eines Processes zu erkennen. Auch in anderer Hinsicht liefern diese Fälle Beiträge für unser Argument, weswegen ich sie hier anfüge, obgleich sie bisher noch nicht veröffentlicht worden sind.

Fall I. Bei einem Syphilitischen im zweiten Jahre der Krankheit, auf dessen Haut sonst keine Manifestationen vorhanden waren, beobachtete ich die Entwicklung von Läsionen, die mir nicht den gewöhnlichen Papeln zu entsprechen schienen. Es fanden sich nämlich unterhalb der inneren Malleolen am Fusse isolirte Krankheitsherde, die einen Durchmesser von $1 / 2 \mathrm{Cm}$. batten, in denen die Haut geröthet und ödematös angeschwollen war; bei der Palpation fühlte man in der tieferen Cutis eine stärkere Resistenz als normal. Eine dieser Läsionen wurde zur Untersuchung ausgeschnitten, während die anderen zur weiteren Beobachtung dienten. Der Verlauf gestaltete sich nun so, dass sie nach 2-3 Wochen verschwanden, ohne dass sie eine sichtbare Veränderung durchmachten. Diese Läsionen unterschieden sich also von den gewöhnlichen Papeln dadurch, dass sich in ihnen nicht Granulomgewebe entwickelte, so dass klinisch das bräunliche Colorit und die derbere Consistenz fehlte.

Auch die histologische Untersuchung bewies, dass es sich nicht um das gewöhnliche Syphiloderm der Frühzeit handelte. In der That aus der Zusammensetzung der Serienschnitte (ca. 300) ergab sich nämlich, dass die Hauptveränderung in einer Phlebitis und Endophlebitis o bliterans bestand, welche fast das ganze im Krankheitsherde liegende Venennet, u.zw. besonders das der Subcutis, betroffen hatte. Die entzündlichen Erscheinungen im Gewebe gruppirten sich um die reränderten Venen. 
Gegenüber diesem Befunde an den Venen ist es wichtig zu bemerken, dass eine Arterie, die den Herd durchzog, unverändert war.

Fall II. Auch hier handelte es sich um einen Syphilitischen im zweiten Jahre der Infection. Bei diesem Kranken beobachtete ich eine diffuse Veränderung der Haut am äusseren Rande des rechten Fusses: sie war leicht geschwollen, bläulich verfärbt und von teigiger Consistenz. Auf dieser Basis entwickelten sich langsam kleine Erhabenheiten, die dann erweichten, aufbrachen und kleine Geschwüre bildeten. Auch hier lagen Veränderungen vor, die von dem gewöhnlichen Typus der Syphilodermen abwichen.

Eine ausgeschnittene Erhabenheit wurde in Serienschnitten untersucht (ca. 200), so dass die Gefässe genau verfolgt werden konnten. Auch in diesem Falle fanden sich ausgebreitete Venenveränderungen, während die Arterien unversehrt waren. Es bestand ebenfalls End o p hle bi t is obliterans der tieferen Cutisvenen und der oberfächlichen Venen der Subcutis,

7. Sull'Eritema e sulla flebite lebbrosa. (Giorn. Ital. mal. ven. e mal. della pelle. 1899.)

Bei vier Leprösen habe ich zu wiederholten Malen acute Ausbrüche der sogenannten hyperämischen Flecke beobachtet, Ausbrüche, die in Wirklichkeit vielgestaltiger sind und auch aus Papeln, Knoten, Infiltraten bestehen können. Da ich bereits früher die oberflächlicheren Formen untersucht hatte, so beschäftigte ich mich dieses Mal mit den tieferen die in aer Subeutis ihren Sitz hatten. In ihrem klinischen Erscheinen sind diese Ausbrüche den idiopathischen Erythemen zu vergleichen und es interessirte mich daher, zu untersuchen, ob sie es auch in histologischen Beziehung sind, um darans neue Beiträge für embolische Veründerungen in der Haut zu gewinnen.

Es stellte sich nun heraus, dass die acuten Veränderungen in der Subeutis mit den bei den ohne bekannte Ursache entstehenden Erythemen vollständig identisch sind, d. h. es besteht eine starke Emigration, hochgradiges sero-fibrinöses Exsudat und auch hämorrhagisches Exsudat. Aber ausser diesen Entzündungserscheinungen kommen bei diesen leprösen Metastasen noch schwerere Veränderungen vor. So habe ich drei Mal Venenthrombose inmitten des Krankheitsherdes gefunden. Die betroffenen Venen wiesen zum Theil nur acute entzündliche Veränderungen ihrer Wandungen auf, zum Theil aber waren sie schon von Leprazellen infiltrirt. In einem vierten Falle schliesslich handelte es sich um Thrombose in einer Vene, deren Wand sehr starke lepröse Infiltration hatte.

Aus diesen Untersuchungen haben wir daher festzuhalten, dass die leprösen Embolien ausser entzündlichen Veränderungen auch Thrombose u. zw. der tiefer gelegenen Venen der Haut hervorrufen können und dass in Folge dieser Localisation des Processes in den Venen sich später eine chronische lepröse Entzündung derselben entwickelt.

Die Fig. 1 und 2 gehören den beschriebenen Fällen an.

8. Ricerche sperimentali sull' Urticaria. (Giorn. Ital. mal. en. e mal. pelle. 1899.) 
Diese besondere Art von Oedem, die nur aus der Dermatologie bekannt ist, wurde bisher auf Nerveneinfluss zurückgeführt, da die für die anderen Arten von Oedem giltigen Bedingungen des Entstehens die charakteristischen Eigenschaften der Urticaria nicht erklärten. Auch war es bisher nicht möglich, dieselbe experimentell zu untersuchen. Da dies mir nun gelungen ist, habe ich ihren Mechanismus genauer feststellen können.

Ich fand eine Reihe von Stoffen, wie Atropiu, Morphium, Pepton, Paraphenylendiamin, mit denen man sowohl auf der menschlichen Haut, wie auf derjenigen von Hunden Urticaria hervorrufen kann. Ich fand ferner, dass es beim Hunde anch nach Eliminirung der sympathischen Nerven, sei es durch Schnitt des Halssympathicus oder durch Ausreissen des oberen Halsganglions oder des ganzen Bauchstranges, möglich ist, experimentell mit den eben angeführten Stoffen Urticaria zu erzeugenUnd schliesslich konute ich mit denselben Stoffen auch durch Injection in das periphere Endo von Arterien in dem ihnen zugehörigen Hautgebiete Urticaria hervorbringen.

Aus diesen Experimenten ziehe ich den Schluss, dass die von innen entstehende Urticaria, wie die von aussen entstehende, durch bestimmte Stoffe erzeugt werden, die eine Gefässreizung bewirken. Die erste Form ist daher den Erythemen gleichzustellen, mit denen sie den Entstehungsmodus theilt, indem nämlich auch bei ihr die patho. genen Stoffe auf dem Blutwege in die Haut gelangen und mit denen sie auch insofern übereinstimmt, als auch sie Gefässwandalteration bewirkt. Die Unterschiede in der Wirkung beider auf die Gefässe lassen sich auf die Art der Ursachen zurückführen: bei den Erythemen haben sie die Eigenschaft, dass sie ausser congestiver Hyperämie und vermehrter Transsudation auch noch Emigration und Austritt von Fibrin bewirken, während bei Urticaria es bei Congestion und vermehrter Transsudation allein bleibt.

\section{Hauptsätze zur Lehre von der Embolie und Metastase in der Haut.}

1. Die erythematösen Hautveränderungen stellen eine besondere Art von Entzündung vor und zwar derjenigen, welchedurchReizeherrorgerufen wird, die auf dem Blutwege in die Haut gelangen.

Die histologischen Befunde, welche man bei den Hautläsionen der als Erytheme bezeichneten Dermatosen macht, sind derartig, dass man sie in pathologisch-anatomischer Sprache entzündliche nennen muss. Man findet nämlich eine mehr oder weniger entwickelte kleinzellige Infiltration und ein mehr oder 
weniger ausgedehntes Oedem, das vorwiegend serös ist, oder einen grossen Gehalt an Fibrin aufweist und auch hämorrhagisch werden kann.

Und wenn wir anatomisch den entzündlichen Charakter zugeben müssen, so werden wir auch in der klinisch erkennbaren Röthe der Läsionen nichts anderes zu sehen haben, als die entzündliche Hyperämie.

Die rerschiedenen Erythemformen, als Flecke, Papeln, Knötchen und Knoten, werden demnach von dem Grade und von dem anatomischen Sitze der Entzündung in der Haut abhängig sein.

Wenn nun die Erytheme weiter nichts als der klinische Ausdruck einer Entzündung sind, so muss es sich doch immerhin um eine Entriundung besonderer Art handeln, die sich wesentlich von der gewöhnlichen Entzündungsform unterscheidet und zwar so sehr, dass ihre Natur bisher vollständig verkannt wurde und viel eher an eine Nervenstörung, Angioneurose, gedacht werden konnte.

Dass dies möglich war, erklärt sich wohl schon daraus, dass man bei Entzündung immer an die in der pathologischen Anatomie gegebene Beschreibung denkt und dann allerdings wird man schwerlich diese Bezeichnung auf einen Process, wie den erythematösen, anwenden, bei welchem der classische Symptomencomplex so wenig ausgebildet ist. Aber andererseits hätte man doch nicht vergessen dürfen, dass auf der Haut wieder unverkennbare Entzïndungsformen vorkommen, bei denen calor und dolor vollständig fehlen können und die deshalb schon zu den erythematösen Hautveränderungen überleiten.

Die gewöhnliche Beschreibung der Entzündung ist nun zwar aus Beobachtungen an der Haut abstrahirt worden, aber sie bezieht sich doch nur auf die hochgradigen Fälle von Entzündung. Bei diesen nunhandelt es sich stets um Erkrankungen, welche durch Ursachen hervorgerufen werden, die direct von aussen aut die Haut einwirken oder die unmittelbar von aussen in die Haut gelangen oder die zwar von innen in die Haut dringen, aber direct von einem unter ihr liegenden Organe, also durch Ursachen, die ihre ihnen eigenthümliche schädigende Wirkung ungeschwächt entfalten können. 
Gelangen aber auf dem Blutwege Entzündungserreger in die Haut, so sind die von ihnen hervorgerufenen Störungen viel milder, weil sie eben entweder ganz anderer Natur sind oder, wenn das nicht, nur in abgeschwächter Form zur Wirkung gelangen können.

Von mechanischen, thermischen und chemischen Ursachen, welche überhaupt nur direct von aussen auf die Körperoberfläche wirken können und hochgradige Entzündung in Gefolge haben, brauchen wir gar nicht za reden.

Aber auch chemische Stoffe, welche von innen aus die Haut schädigen, können nicht mehr eine so starke Wirkung ausüben, wie wenn sie direct von aussen zur Geltung kommen, weil sie schon, beror sie die Haut treffen, vielfältig Gelegenheit haben, ihre Affinitäten zu sättigen.

Und die Mikroorganismen, deren Virulenz wir daran messen können, dass sie die Haut stark schädigen, wenn sie von aussen auf dieselbe gelangen, zeigen wesentlich abgeschwächte Wirkung, wenn sie auf dem Blutwege dahin transportirt werden.

Ausserdem wissen wir, dass bei den Entzündungen der Haut aus inneren Ursachen auch Stoffe in Frage kommen, die im Innern des Körpers selbst gebildet werden und daher gewiss nicht so stark entzündungserregende Eigenschaften besitzen werden, wie die gewöhnlichen chemischen Substanzen.

Wenn wir diese Verhältnisse berücksichtigen, so können wir sehr gut verstehen, dass der vorschiedene Entstehungsmodus der Entzündung einen Unterschied zwischen ihrer gewöhnlichen Form und der erythematösen oder hämatogenen Form bedingt.

Aber ausser dem Unterschiede in dem Grade der Symptome, welcher an eine principielle Verschiedenheit zwischen Entzündungen und Erythemen denken liess, scheint auch ein anderer Umstand die richtige Erkenntniss erschwert zu haben, nämlich der Umstand, dass auf der Haut die Entzündung zumeist von Oberhautveränderungen begleitet ist, die bei den Erythemen sehr zurücktreten.

Bei den sogenannten Dermatitiden sind die Epidermisalterationen so gewöhnlich, dass man sie deswegen seit Auspitz direct als Katarrhe bezeichnet, weil entweder Ahschuppung stattfindet oder sich ein feuchtes Exsudat auf der Oberfläche 
bildet, das ebenfalls von pathologischen Störungen der Epidermiszellen abhängig ist. Und diese Oberhautveränderungen gehen den entzündlichen Cutisveränderungen parallel. Anders bei den Erythemen. Die Blutkreislaufstörungen können hier ganz allein bestehen, ohne jegliche Oberhautstörung. Das fällt besonders da auf, wo starke erythematöse Läsionen vorliegen, wie beim Erythema nodosum, wo sich trotzdem an der Epidermis keine Anomalien entwickeln. Zwar kommen nun auch bei den Erythemen Epidermisveränderungen vor, nämlich die Blasen, aber diese sind doch so eigenartig, dass sie nicht mit den entzündlichen Epidermisveränderungen zusammengeworfen werden können. Daher konnten sie auch nicht dazu dienen, über den wahren Charakter der Erytheme aufzuklären.

Wenn man aber der von mir aufgestellten Unterscheidung zwischen der gewöhnlichen Entzündung und der erythematösen als bämatogenen folgt, so versteht man auch diese Differenzen sehr gut.

Wirken nämlich die Entzündungserreger von aussen, so treffen sie zuerst die Epidermis und dann die Blutgefässe der Cutis; die darauf folgende Entzündung wird daher von Epidermisveränderungen begleitet sein. Dringen sie dagegen auf dem Blutwege in die Haut, so kommt zunächst die Wirkung auf die Gefässe zur Geltung, d. h. es bildet sich Entzündung ohne Oberhautläsionen aus. Ob diese sich dann später noch ausbilden werden, hängt von der Weiterentwicklung des Processes ab. Jedenfalls sind sie nicht so direct und primär entstanden, wie bei der ersten Form der Entzündung.

Der Auffassung der Erytheme als Entzündungen stehen daher auch rom klinischen Standpunkte aus keine Hindernisse im Wege. Ihre Eigenschaften erklären sich sehr gut aus der Art ihres Enstehens.

Wir können daher nun der gewöhnlichen Form der Entzündung, als exogenen Entzündung, die auf dem Blutwege entstandene gegenüberstellen und sie kurz hämatogene Entzündung nennen.

Eine allgemein pathologische Beschreibung dieser Art von Entzündung lässt sich demnach leicht auf Grund der klinischen Darstellung der Erytheme geben. 
Dieselbe besteht klinisch aus congestiver Hyperämie mit oder ohne Oedem. Diese Hyperämie unterscheidet sich ron der gewöhnlichen entzündlichen Hyperämie durch die schnelle Entwicklung und ihren milderen Verlauf. Auch das Oedem zeichnet sich durch sein schnelles Auftreten aus und dadurch, dass es im Verhältniss zur Congestion stark ausgebildet ist und sich auch stärker entwickelt, als es sich bei gleichem Grade von entzündlicher Hyperämie entwickeln würrde.

Ferner fehlen zumeist primäre Epidermisveränderungen und, wo sie vorhanden, bestehen sie aus eigenthümlichen blasigen Abhebungen.

So stellt sich denn in der Klinik die bämatogene Entzündung in einer ganz besonderen, von der exogenen Entzïndung sehr unterschiedlichen Form dar.

Ihrem anatomischen Sitze nach, kann man eine oberflächliche und eine tiefe hämatogene Entzündung unterscheiden, von welchen beiden gerade die letztere sehr charakteristisch ist. Denn bei der gewöhnlichen Entzündung beginnt der Process an der Oberfläche, um allmälig in die Tiefe zu gelangen, während hier schon primär die Fntzündung ihren Sitz in den tieferen Lagen der Haut baben kann.

Was den weiteren Verlauf der hämatogenen Entzündung betrifft, so bleibt sie der Regel nach als solche bis zum Erlöschen bestehen, sie bildet nicht so häufig, wie die gewöhnliche Entzündung, nur den einleitenden Process für darauf folgende schwerere Gewebsstörungen, wie Eiterung, Necrose, Geschwürsbildung etc.

Mit diesen Worten glaube ich, die Hauptunterschiede zwischen den beiden Entzündungsformen hervorgehoben zu haben und damit auch die neu aufgestellte charakterisirt $z u$ haben.

Wenn wir nun von diesem neven Gesichtspunkte aus solche Processe in Betracht ziehen, die entzündlicher Natur sind und bei denen wir einen hämatogenen Entstehungsmodus kennen oder mit Recht vermuthen, so werden wir auch hier der bämatogenen Entzündung begegnen müssen.

Wir beobachten sie daher vor allem bei bakteriellen Embolieen in die Haut, wie bei pyämischen und septischen Processen, bei Lepra, wo die Wirkung der Mikroorganismen, 
beziehentlich ihrer Toxine, allein in Gefässreizung besteht oder wo sich an diese auch weitere Veränderungen anschliessen können, sei es Eiterung oder, was gewöhnlicher ist, Granulombildung.

Auch in meinem Falle von tu ber cu lös er Embolie traten die Herde in der Haut in entzündlicher Form auf und verliefen entweder ohne weitere Veränderungen durchzumachen, oder es schloss sich an die Entzündung Eiterung und Necrose an.

Bei Syphilis liegt den verschiedenen Arten ron Roseola und Erythemen ebenfalls die hämatogene Entzündung zu Grunde. Aber auch bei den verschiedenartigen Papeln hat die im Beginne sichtbare Röthe dieselbe Bedeutung.

2. Der klinische Verlauf der erythematösen. Hautveränderungen stellt den Typus der auf dem Blutwege entstandenen Metastase ror.

Wenn wir in den anatomischen Veränderungen der einzelnen Erythemläsion nichts weiter als die hämatogene Form der Entzündung sehen, so haben die Erythemläsionen als Ganzes genommen folgerichtig die Bedeutung der allgemeinen Form, unter welcher Blutgefässmetastasen in der Haut auftreten.

Die Beschreibung, welche die Lehrbücher in dem Capitel Erytheme von der Art ihres Auftretens, ihrer Localisation, ihrer Entwicklung, ihrer Ausbreitung in der Haut geben, beziebt sich daher nicht mebr auf bestimmte Krankheiten oder auf eine besondere Krankheitsgruppe, sondern diese Eigenschaften kommen ihnen deshalb zu, weil sie ein und dieselbe Pathogenese haben, weil sie nämlich auf dem Blutwege entstanden sind.

Aus der von ihnen gegebenen klinischen Beschreibung können wir daher ohne weiteres die allgemein pathologische Schilderung der hämatogenen Metastasenbildung abstrahiren.

Dieselbe würde danach ungefähr so zu lauten haben:

Gelangen mit dem Blute entzündungserregende Stoffe in die Haut, so entwickeln sich in kurzer Zeit über die ganze Körperoberfläche vertheilte Entzündungsherde. Diese treten entweder in diffuser Form auf und befallen ganze Regionen oder erscheinen in kleiner umschriebener Form, isolirt inmitten gesunder Haut.

Die Vertheilung dieser Herde ist meist eine symmetrische. 
Die isolirt auftretenden Herde können einen verschiedenen anatomischen Sitz haben: sie finden sich entweder in der oberflächlicben Schicht der Cutis oder in den tieferen Lagen oder auch von Anfang an selbst in dem Unterhautzellgewebe.

Ebenso bemerkenswerth wie die Schnelligkeit, mit welcher: zu gleicher Zeit an verschiedenen Körperstellen diese Krankheitsherde erscheinen, ist auch die Schnelligkeit, mit welcher sich der einzelne Herd entwickelt und sein Höhestadiura erreicht, das meist jedoch nicht über die einfache entzündliche Störung hinausgeht.

Das acute, gleichzeitige Auftreten von vielen Krankheitsherden, das Ausbruch, Eruption, Schub genannt wird, kann sich bei einem Krankheitsfalle häufiger wiederholen und bestehen dann $\mathrm{zu}$ einer gegebenen Zeit auf der Haut Herde nebeneinander, die sich in verschiedenen Phasen der Entzündung befinden.

Manche dieser Eigenschaften nun, wie die Schnelligkeit des Auftretens und der Verbreitung und die Symmetrie, mögen zwar leicht dazu fuihren, in den Erythemen nervöse Störungen zu sehen, aber doch ist es nicht zu verkennen, dass die von mir vertheidigte Auffassung eine genügende Erklärung von diesen Erscheinungen gibt. Denn bei embolischen Processen gelangen die pathogenen Stoffe sehr schnell in die verschiedenen Theile eines Organes, wie der Haut, und können symmetrisch abgelagert werden. In dieser Hinsicht also leistet meine Theorie zumindest dasselbe, wie die Angioneurosenlehre, und ersetzt sie daher auch auf diesem Gebiete.

Ausser den bisher besprochenen Eigenschaften kommt der hämatogenen Metastase auch eine bestimmte Topographie zu.

3. Den hämatogenen Metastasen in der Haut kommt eine bestimmte Topographie zu.

Von embolischen Processen im Innern des Körpers wissen wir, dass sie gewisse Organe besonders häufig befallen, während andere davon mehr verschont bleiben. Ausser von den Ursachen dieser Processe hängt dieses Verhalten auch von der anatomischen Structur der betreffenden Organe ab. Für die Localisation grösserer Embolie ferner ist häufig der Bau des Aortensystemes von bestimmendem Einfluss, so zwar, dass sie leicht in denjenigen Arterien 
stecken bleiben, welche die geraden Fortsetzungen der Aorta nach oben und nach unten bilden, $d . h$. in den Art. fossae sylvii und in den Art. saphena.

Es scheinen nun ähnliche Verhältnisse auch bei den embolischen Processen der Haut obzuwalten, in dem Sinne nämlich, dass gewisse Regionen der Körperoberfläche häufiger Sitz von Metastasen sind als andere.

Eine derartige Deutung wenigstens können wir, unserer allgemeinen Auffassung von Erythemen gemäss, der klinischen Beschreibung geben, wonach die typische Localisation des Erythema multiforme die Streckseiten der Extremitäten sind. Mag man dieses Hebra'sche Krankheitsbild in dem von ihrem Autor gemeinten Sinne auffassen oder es überhaupt nur als klinische Symptomengruppe gelten lassen, es ist eine leicht zu beobachtende Thatsache, dass bei den Erythemen häufiger die Extremitäten als der Rumpf befallen werden, dass sie ausserdem frühzeitiger betroffen werden und dass an den Extremitäten es wiederum die Streckseiten sind, die stärker erkranken, als die Beugeseiten.

Wir können demnach die Extremitäten und besonders ihre Streckseiten geradezu als das Praedilectionsgebiet der bämatogenen Metastase bezeichnen.

Wenn dem wirklich so ist, so dürfen wir erwarten, dasselbe auch von anderen ähnlichen Processen respectirt zu sehen. In der That begegnen wir denn auch bei der Lepra einer gleichen Bevorzugung der Extremitäten vor dem Rumpfe. Dies geht schon, ohne dass ich mich auf eigene Beobachtung bei den acuten Ausbrüchen von Lepraerythemen zu berufen brauche, aus der Beschreibung hervor, welche Danielssen und Boeck und Leloir davon geben.

"L'éruption des taches se montre tantôt au visage, tantôt aux extrémités, tantôt sur tout le corps. Cependant les taches affectent des sièges de prédilection: le masque facial; ce sont les membres supérieurs et inférieurs, surtout du côté de l'extension, la face dorsale des mains. On les trouve aussi dans le dos trèssouvent et en grande quantité aux fesses."

Auch in dem von mir beschriebenen Fall von $t u b$ e r culöser Metastase in der Haut waren hauptsächlich die Beine und 
Fuisse und die Arme und Hände Sitz der Krankheitsherde. Eine gleiche Localisation finde ich ferner auch in jenen unter verschiedenen Namen beschriebenen Fällen (tuberculides) angegeben, bei denen ich, wie ich bereits oben gesagt habe, eine dem meinigen ähnliche Pathogenese vermuthe.

Ebenfalls bei S y p hil is kommt die besprochene Praedilection vor, denn die beiden Secundärläsionen, die ich beschrieben habe, hatten ihren Sitz an den Füssen und rom Gumma ist es ja bekannt, dass es sich mit Vorliebe an den Beinen, Armen und am Kopfe localisirt.

Was demnach auch der Grund dafür sein mag und mögen auch wichtige Ausnahmen dabei vorkommen, es scheint mir doch eine Regel zu sein, dass es auf der Haut bestimmte Regionen gibt, an denen früher und häufiger die hämatogenen Metastasen sich entwickeln als auf der übrigen Körperoberfläche.

Das Befallensein gerade dieser Regionen in einem gegebenen Krankheitsfall dürfte daher mit als Erkennungszeichen seiner Pathogenese gelten können.

4. Die hämatogene Entzündung localisirt sich häufig an den Venen.

Die classische Darstellung der Embolie und ihrer Folgen, welche sich hauptsächlich auf das Schicksal gröberer corpusculärer Elemente, die dem Blute beigemengt sind, bezieht, gilt bekanntlich auch für feinere Elemente, insbesondere auch für Mikroorganismen, die ursprünglich in jener Lehre ja gar nicht vorgesehen waren. Auch von diesen weiss man, dass sie, einmal in den Kreislauf gelangt, in die kleinsten Arterien und Capillaren verschleppt werden und dort ebenfalls stecken bleiben können, wie die amorphen Stoffe.

Auch in der Haut kommt unzweifelhaft diese typische Form der Embolie vor, in welchem Falle wir dann in den Arteriolen und in den Capillaren die Mikroorganismen vorfinden. So habe ich selbst bei den acuten Ausbrüchen der Lepra in den frisch entstandenen Flecken der Haut die Bacillen in den kleinen Arterien und den Capillaren auffinden kömen.

Aber im allgemeinen gelten für die bacilläre Embolie nicht dieselben Regeln, wie für diejenige von gröberen Stoffen, aus dem einfachen Grunde, weil für die feinen Bakterien die Enge 
der Gefässe nicht so leicht als mechanisches Hinderniss zur Geltung kommt. Wir wissen daher auch, dass sie sehr gut nicht allein die Endverzweigungen der Arterien, sondern auch die Capillaren ungehindert passiren können. Und wenn trotzdem die Bakterien, besonders in den Capillaren gefunden werden, so müssen für dieses Liegenbleiben andere Umstände vorwalten, wie z. B. die Verlangsamung des Blutstromes oder Gefässwandveränderungen oder Gerinnungen des Blutes.

Die classische Lehre von der Embolie stellt sich demnach für die Mikroorganismen als nicht ausreichend heraus, weil eben die mechanischen Momente bei ihnen eine viel geringere Rolle spielen.

Und womöglich noch unzureichender erweist sie sich, wenn es sich überhaupt nicht um morphologische Elemente handelt, die vom Blutstrom fortgetragen werden, sondern um im Blute gelöste Stoffe, für die natürlich mechanische Factoren nicht in Frage kommen können. Die Wirkung dieser könnte a priori in jedem beliebigen Abschnitt des Gefässsystemes, sowohl an den Arterien, wie an den Venen und an den Capillaren zur Geltung kommen.

Wenn ich nun das Resultat meiner diesbezüglichen Untersuchungen bei embolischen Processen der Haut zusammenfasse, so ergibt sich als eine neue Thatsache, dass es nicht der arterielle Gefässabschnitt ist, der hiebei hauptsächlich betheiligt ist, sondern vielmehr der venöse.

Dies tritt besonders dann hervor, wenn es sich um schwerere Störungen handelt, wie Thrombose, Stasis und Gefässwandveränderungen, die ich nämlich zumeist gerade an den Venen und nicht an den Arterien beobachtet habe.

Ich beziehe mich hier auf die Befunde, welche ich bei den acuten Erythemausbrïchen der Lepra gemacht habe. In den Fällen, wo hiebei Thrombose vorlag, waren es Venen, die befallen waren. Auch die chronische lepröse Gefässwandentzündung fand ich desgleichen in ihnen localisirt.

Ich führe weiter den Fall von $t u b$ e r culö ser Metastase in der Haut an, bei welchem ebenfalls in typischer Weise, es stets die Venen waren, in denen Thrombose rorkam. Auch habe ich zweimal, wo Tuberkelbacillen in den Präparaten ge- 
funden wurden, dieselben nur im Innern und in den Wandungen von Venen und nicht ron Arterien gesehen.

Ferner gehören hierher die Befunde von primären Gefässveränderungen, die gewissen secundären und tertiären S y philodermen zu Grunde liegen und bei denen es sich in der überwiegenden Mehrzahl der Fälle stets nur um Venenreränderwngen u. zw. um Endophlebitis obliterans handelte.

Wie man sieht, bin ich durch Untersuchung des verschiedenartigsten Materials immer wieder auf die Venen hingewiesen worden, so dass ich mir schliesslich habe sagen müssen, dass es sich hier, wenigstens für die Haut, um eine allgemeine Regel handeln dürfte, nach welcher nämlich die Venen leichter dem Einfluss schädigender Stoffe unterliegen, als die Arterien.

Und wenn ich nun danach suche, ob bereits schon ein derartiges Verhältniss in der Pathologie bekannt ist, so glaube ich in folgenden beiden Thatsachen eine Bestätigung meines Satzes finden zu können.

Wie aus den Beschreibungen der unter dem Mikroskop angestellten Entzündungsversuchen hervorgeht, beginnt die Emigration an den Capillaren und an den Venen und erst später erfolgt sie auch an den Arterien. Dieses verschiedene Verhalten zwischen Venen und Arterien findet man nun auch in der menschlichen Haut bestätigt, weniger deutlich bei Entzündung in der Cutis, aber sehr klar bei der hämatogenen Entzündung der Subcutis, wo die Gefässe getrennt in einem lockeren Gewebe liegen und daher leicht gesondert beobachtet werden können. Beispielsweise beim Erythema nodosum kann man häufig Vene und Arterie nebeneinander verlaufend finden, ron denen die erstere von weissen Blutkörperchen umgeben ist, während letztere davon noch so gut wie frei ist.

Und die zweite Thatsache, die mir für eine grössere Vulnerabilität der Venen gegenüber den Arterien zu sprechen scheint, ist die, dass gerade Venenthrombose im Anschlinss an Infectionskrankheiten auftritt. Wie man weiss, kommt bei Typhus, Scharlach, Pocken, Puerperalfieber, Cholera u. a. m. nicht so selten Thrombose der grösseren Venen, meistens an den Beinen, vor. Ueber ihre Ursache ist man zwar noch nicht im Klaren und sucht dieselbe theils in Intimaveränderungen, theils in der 
Zusammensetzung des Blutes selbst. Wie dem auch sein mag, für uns genügt die Thatsache selbst.

Wie hier acute Infectionen massgebend für Venenveränderungen sind, so sind auch solche bei chronischen Infectionskrankheiten aus der Klinik bekannt. So kennt man Venenentzündungen im Verlaufe der Syphilis, wie auch im Verlaufe der Lepra, auf welche neuerdings Gl ï ck auf dem Intern. Congress für Lepra die Aufmerksamkeit gelenkt hat. Und zwar handelt es sich hier, worauf es uns augenblicklich ankommt, stets um primäre Gefässalterationen und nicht um solche, die durch Weiterverbreitung eines schon vorher im umgebenden Gewebe bestehenden Processes verursacht werden.

Es gibt also auch klinische Thatsachen, welche für eine besondere Disposition der Venen, auf Entzündungsreize zu reagiren, sprechen.

Bei diesen klinisch wahrnehmbaren primären Venenthrombosen und Venenentzündungen haben wir nun im grossen denselben Vorgang vor uns, der sich im kleinen, in isolirten Krankheitsherden der Haut, in sogenannten Efflorescenzen, abspielt.

Bei den Erythemknoten, die im Verlaufe ron Infectionskrankheiten auftreten, können demnach primäre Venenthrombose und primäre Venenentzïndung vorliegen, wie wir es bei Lepra, Tuberculose und Syphilis gefunden haben. Und selbst das scheinbar weit abliegende Gumma der syphilis stellt in diesem Simne nichts anderes als eine primäre Venenentzündung vor.

Für die klinisch-anatomische Diagnose ist es daher von Wichtigkeit, bei den hämatogenen Entzïndungsprocessen, besonders wenn sie in den tieferen Cutisschichten oder gar in der Subcutis localisirt sind, an die Möglichkeit zu denken, dass Venenthrombose oder Venenentzündung vorliegt.

Als Belege für die besprochene primäre Venenentzündung, die in isolirten Krankheitsherden in der Haut auftritt, scheint es mir angebracht, anhangsweise einige histologische Präparate bildlich wiederzugeben.

Es handelt sich hier stets um Läsionen, die in Serienschnitten (Paraffineinbettung, Schnittdicke 10-15 $\mu$ ) untersucht wurden und bei denen der Gefässverlauf und das Verhältniss der Gefässe zu den Gewebsveränderungen genau reconstruirt wurde. Nur auf diese Weise lässt sich mit Sicherheit feststellen, dass die Gefässveränderungen wirklich primäre sind and den Ausgangspunkt für etwaige später sich entwickelnde pathologische Vorgänge im umliegenden Gewebe bilden. 
Fig. 1. Acut entstandenes erythematöses Knötchen vom Arme eines Falles von Lepra tuberosum, beschrieben in der Pablication Nr. 7.

Das bis in die Subcutis ausgeschnittene Stück wurde in c. 192 horizontale Schnitte zerlegt. Der Durchmesser derselben beträgt $5 \mathrm{Mm}$, wäbrend der im Centrum gelegene Herd nur 2-3 Mm. misst. Die wichtigsten Veränderungen finden sich durch ea. 80 Schnitte hindurchgehend. Inmitten des normalen Fettgewebes besteht ein schon ausgebildetes lepröses Gewebe, durch welches die Fettzellen ersetzt sind. Dieser ältere Herd umgibt nun verschiedene Venen, an denen man folgende frische Veränderungen wahrnimmt. Eine grössere horizontal verlaufende Vene (Durchmesser 0.122 Mm.) ist durch Stasis erweitert, wandständig sieht man Leukocyten, die an den Einmündungsstellen von Aesten dieses Gefässes Thromben bilden. Um die erweiterte Hauptvene findet man freie rothe Blutkörperchen und durch Stasis ausgedebnte Capillaren. Die von oben in sie einmündenden Aeste werden nach ihrem peripheren Ende zu normal. An der Peripherie des eben beschriebenen Herdes verläuft eine normale Arterie.

Diagnose: Acute lepröse Venenentzündung.

Fig. 1 a ist ein Schema des erkrankten Venennetzes.

Fig. $1 \mathrm{~b}$ ist ein Schnitt aus der im Schema angegebenen Höhe: im Cenirum die durch rothe Blutkörperchen erweiterte Vene mit dem Uebergang der von oben kommenden Aeste in sie; in der normalen Peripherie des Schnittes eine Arterie.

Fig. $1 \mathrm{c}$ ist ein um ca. 7 Schnitte höher gelegenes Präparat, worin die Durchschnitte der in $b$ sichtbaren Aeste wiederzusehen sind. Hier aber ist ihr Lumen durch weisse Blutkörperchen ausgefüllt.

Fig. 2. Acut entstandener subeutaner Knoten vom Beine eines Falles von gemischter Lepra. Knoten in 160 Horizontalschnitte zerlegt, deren Durchmesser ca. I Cm. beträgt, beschrieben in der Publication Nr. 7.

Schon makroskopiscb lässt sich in den Scbnitten inmitten des Fettgewebes durch das ganze Stück ein Gefäss verfolgen, das einen Durchmesser von $1 \mathrm{Mm}$. hat. Dieses Gefäss erweist sich als eine Vene, deren Adventitia von Leprazellen infiltrirt ist.

Während in allen anderen Schnitten ihr Lumen offen ist, finden sich in ea. 20 Schnitten folgende Veränderungen: das Lumen ist mit Leukocyten ausgefüllt, die Venenwand ist an dieser Stelle stark ödematös und von Rundzellen infiltrirt, so dass hier der Durchmesser des Gefässes grösser wird, als vor und hinter diesem Punkte. Von der Vene aus gehen diese frischen Veränderungen in das umliegende Bindegewebe über, in welchem man fibrinöses Exsudat und Rundzelleninfiltrat findet.

Das umgebende Fettgewebe ist unverändert, bis auf einige kleine Herde von Leprazellen.

Diagnose: Acute Entzündung und Thrombose in einer leprös infiltrirten Vene.

Die Figur gibt einen der 20 Schnitte wieder, um die acuten Veränderungen zu zeigen. 
Fig. 3 und 4 gehören dem Falle von tuberculöser Phlebitis an, beschrieben in der Publication $\mathrm{Nr} .5$.

Fig. 3 stammt von einem $1 \mathrm{Tag}$ alten, bis in die Subcutis reichenden erythematösen Knötchen des Oberschenkels. Es ist ein senkrecht zur Hautfäche geführter Schnitt, in dem man dicht unter der Cutis eine Vene sieht, deren Lumen von Leukocyten und Fibrin ausgefüllt ist. In der Venenwand und im umliegenden Gewebe bestehen nur geringe Veränderungen, als $0 e d e m$ und kleinzellige Ansammlungen.

Fig. 4. stellt das Endstadium desselben Processes dar, nämlich ein vereitertes Knötchen von der Wade. Aber auch hier kann man noch in Serienschnitten die primäre Phlebitis und den Zusammenhang des Abscesses mit ihr nachweisen.

Das Knötchen, das einen Durchmesser von $8 \mathrm{Mm}$. in der Breite und von $6 \mathrm{Mm}$. in der Tiefe hatte, wurde in 175 Schnitte zerlegt.

Durch das ganze Stück lässt sich dicht unter der Cutis eine Vene verfolgen, die thrombosirt ist. Am Rande des Stückes finden sich in ihrem Lumen ausser weissen Blutkörperchen auch noch rothe und ihre Wand ist nicht verändert. Je päher man aber dem Centrum des Krankheitsherdes kommt, um so grösser wird ihr Lumen und um so stärker wird die Anschwellung ihrer Wand, bis schliesslich Venenwand und Thrombus zu einer amorphen Masse verschmelzen, in der nur noch Kernreste zu erkennen sind.

In diese Hauptvene münden nun cutane Aeste, die zum Theil throm bosirt sind und zum Theil als necrotisch aufzufinden sind, in letzterem Falle kenntlich an dem durch Orcein färbbaren und noch persistirenden elastischen Gewebe ihrer Wand.

Um die Hauptvene sieht man im Fettgewebe hie und da Inseln von Riesenzellen.

Fig. 4a. In der Subcutis befindet sich ein Querschnitt der Vene, in deren Lichtung Leukocyten und rothe Blutkörperchen liegen. In der Umgebung sieht man ein kleinzelliges Infiltrat und Herde von grösseren Zellen mit einzelnen Riesenzellen.

In der Cutis bestehen Rundzellenherde um die Gefässe. Dieser Schnitt ist von der Peripherie des Stückes. entfernt.

Fig. 4b. Dieses Präparat ist um 60 Schnitte von dem vorigen

Die Vene in der Subcutis ist schräg und zweimal getroffen; ihr Lumen ist thrombosirt und die kleinzellige Infiltration ist stärker geworden.

Auch in der Cutis ist die Entzündung stärker; aus ihrer strichförmigen Anordnung kann man schon erkennen, dass sie perivasal ist, was bei stärkerer Vergrösserung leicht $\mathrm{zu}$ bestätigen ist, u. zw. handelt es sich um phlebitische Herde.

Fig. 4c. Hier sind wir noch um 90 Schnitte weiter und im Centrum des Krankheitsherdes. Was zuerst auffällt, ist der cutane Abscess, von dessen Entstehung aus phlebitischen Herden ich oben schon gesprochen habe. 
Die suhcutane Vene ist quer getroffen und als Kreis mit peripherer radiärer Schrafirung sichtbar. Ihr Thrombus und ihre Wand sind schon nekrotisch.

Fig. 5. Syphilitisches Gumma vom Arme eines Mannes (siehe pag. 47). Dasselbe hatte einen Durchmesser von $1 \mathrm{Cm}$. u. wurde in 140 Horizontalschnitte zerlegt. Die Schnitte hatten einen Durchmesser von $8 \mathrm{Mm}$., der centrale Krankheitsherd aber nur von 3-4 Mm. Schon mit schwacher Vergrösserung sieht man durch das ganze Stück von oben nach uuten eine Vene ziehen. Sie geht von einer normalen unter der Cutis liegenden Vene aus und tritt dann in den Granulomberd mit erhaltenen Zellen ein. Hier ist ihre Wandung kleinzellig infiltrirt und ihr Lumen leer und enge. Tarauf wird sie nekrotisch, bleibt aber kenntlich an den erhaltenen elastischen Fasern ihrer Wand, während die zelligen Elemente verschwunden sind. An dem von den elastischen Fasern begrenzten Raum erkennt man, dass ihr Iumen, das stark erweitert ist, von nekrotischer Masse ausgefüllt ist. Das umgebende Gewebe ist ebenfalls in eine amorphe Masse verwandelt Die Vene verlässt schliesslich diesen Herd, nimmt wieder normale Dimensionen an, enthält zuerst noch Rundzellen im Lumen, dann Spindelzellen und im weiteren Verlauf nacbeinander Fibrin und Blut, um wieder ganz normal $\mathrm{zu}$ werden.

Diagnose: Endophlebitis proliferans sifilitica mit consecutiver Necrose.

Fig. 5a ist ein Schema des Verlaufes der erkrankten Vene und ihres Verhältnisses zu dem Granulom. Die horizontalen Linien deuten die Höhe an, aus welcher die beiden folgenden Schnitte genommen wurden.

Fig. $5 \mathrm{~b}$. Inmitten des wenig infiltrirten Fettgewebes sieht man zwei structurlose Flächen, die dem nekrotischen Granulomgewebe entsprechen. Tangential zu dem centralen Herde verläuft die Vene, die peripherwärts normal ist, dann aber breiter wird, im Lumen spindelförmige Zellen (und eine Riesenzelle) enthält und eine infiltrirte Wand zeigt.

Fig. $5 \mathrm{c}$, um 24 Schnitte von dem vorigen entfernt, lässt inmitten einer amorphen Masse die quer getroffene Vene sehen, deren Lumen durch die nekrotisch gewordenen Zellen erweitert ist und von deren nekrotisch gewordenen Wandelementen nur noch das elastische (durch Orcein) gefärbte Fasernetz erhalten ist.

Fig. 6. Syphilitisches Gumma vom Arme eines jungen Mädchens (siehe pag. 17). Das ausgeschnittene Stück hatte in den Schnitten einen Durchmesser von 6-7 Mm., der darin befindliche Krankheitsherd einen von 2-3 Mm. 189 Horizontalschnitte.

Die ganze Veränderung stellt sich wie ein periphlebitischer Herd dar. Von einer normalen, senkrecht durch das Stück ziehenden Vene geht ein schräg durch dasselbe laufender Ast ab, der zuerst erkrankt ist und später wieder normal wird.

Die Veränderungen der Vene selbst und ihr Verhältniss zu dem Granulom sind dem vorigen Falle so gut wie gleich, weswegen ich auf 
eine detaillirtere Beschreibung verzichten und mich auf eine Erläuterung der Figuren beschränken kann.

Fig. 6a. Schematischer Verlauf der im Stück enthaltenen Venen. Die punktirte Linie um die kranke Vene gibt die Grenze des Granuloms an. Anch ist eine normale Arterie angegeben, die ausserhalb des Krankheitsherdes verläuft.

Die beiden Horizontallinien entsprechen den beiden folgenden Figuren.

Fig. 6 b. Inmitten des wenig infiltrirten Fettgewebes sieht man eine schräg getroffene Vene, deren peripheres Ende fast normal ist, denn es finden sich nur Leukocyten im Lumen. Ihr centrales Ende ist wesentlich verändert: ihr Lumen ist von Spindelzellen ausgefüllt und ihre Wandung ist nicht gut von der Umgebung abzugrenzen. Ihre zelligen Elemente sind nämlich nekrotisch geworden und sind nur noch die elastischen Fasern erbalten. Das umgebende Gewebe ist amorph und hie und da findet man Rundzellenherde.

Fig. 6c. Dieses Präparat ist um 20 Schnitte von dem vorigen entfernt.

Im Centrum des Schnittes sieht man inmitten einer amorphen Masse die gänzlich nekrotisch gewordene Vene, die nur an dem elastischen Netz kenntlich ist. In der normalen Peripherie des Schnittes ist der Querschnitt einer grossen normalen Vene an der einen Seite und der einer normalen Arterie an der anderen.

5. Die Urticarialäsion beruht auf einerGefässwandalteration und die Urticariakrankheit ist ein embolischer Process.

Haben wir erst einmal erkannt, dass die erythematösen Hautveränderungen nichts anderes als auf dem Blutwege entstandene Entzündungen sind und nicht, wie es gelehrt wird, angioneurotische Störungen, so werden wir schliesslich auch eine andere Hautläsion von diesem neuen Gesichtspunkte aus betrachten müssen, nämlich die Urticaria.

Dies habe ich in der oben angeführten Arbeit gethan und bin ich darin auf experimentellem Wege zu dem Schlusse gelangt, dass die von innen aus entstehende Urticaria auf einer directen Reizung der Gefässwand beruht, welche durch ins Blut gelangte Stoffe ganz bestimmter Natur ausgeübt wird.

Damit stelle ich von anatomischem und pathogenetischem Standpunkte aus die Urticaria neben die Erytheme. Gemeinsam mit diesen hat sie nämlich, dass auch bei ihr die pathogenen Stoffe auf dem Blutwege in die Haut gelangen und dass diese auch bei ihr active Hyperämie und seröses Transsudat hervor- 
rufen. Die Unterschiede, welche zwischen den beiden Processen bestehen, hängen von der Ursache $a b$ und sind im wesentlichen nur Gradunterschiede.

Die Urticaria verläuft schneller, weil die Wirkung der reizenden Stoffe schneller paralysirt wird und die Natur derselben es mit sich bringt, dass sie weniger schädigend auf die Gefässwand einwirken, als es bei den Erythemen der Fall ist.

Damit hängen auch die histologischen Differenzen zusammen, dass nämlich bei den Erythemen Auswanderung von Leukocyten stattfindet und das Transsudat Fibrin enthält, Befunde, die der Urticaria abgehen. Aber andererseits gibt es in dieser Hinsicht bereits sehr grosse Unterschiede selbst unter den verschiedenen Formen von Erythemeu, die dem Grade der Emigration und in dem Gehalte an Fibrin nach ausserordentlich variiren, so dass ein allmäliger Uebergang von ihnen zur Urticaria zu beobachten ist.

Aber auch in klinischer Hinsicht sind Uebergänge zwischen erythematösen und urticariellen Hautveränderugen unverkennbar.

Zwar wird in den Lehrbuichern Urticaria als besondere Krankheit abgehandelt, doch stellt sie im Grunde nichts weiter als eine einfache Hautläsion vor, als welche sie übrigens schon von der französischen Schule erkannt worden ist, die in der Urticaria nur ein Symptom der verschiedensten Krankheiten sieht.

Aber ich möchte noch einen Schritt weitergehen und sagen, dass auch die Efflorescenz Urticaria die sogen. Quaddel an und für sich gar keine besondere, für sich allein dastehende Elementarform ist, der als solcher ein Platz neben den anderen eingeräumt werden muss. Denn morphologisch kann sie gewissen bei Erythemen vorkommenden Läsionen vollständig gleich sein und andererseits sind die bei ausgebreiteter Urticaria vorhandenen Elementarformen nicht immer gleich und können grosse Unterschiede in Bezug auf Umfang, Form, Farbe und Sitz aufweisen. Es wurde eben als Typus für die Quaddel, die durch Urtica hervorgebrachte Hautveränderung angenommen und dieser Name dann auf andere nur ähnliche Läsionen übertragen, wie es so häufig in der Dermatologie auch bei anderen Eiflorescenzen gemacht worden ist. 
Aber lassen wir jetzt diese Frage der Morphologie als von nebensächlicher Bedeutung bei Seite und halten wir uns vielmebr nur an die klinisch-anatomischen Eigenschaften! Alsdann können wir den bei der Urticaria vorliegenden Process als flüchtiges Oedem mit Hyperämie oder flüchtiges Reizödem bezeichnen, dem der hämatogene Entzündungsprocess der Erytheme gegenüberstebt.

Ich sage nun, dass diese beiden Arten von Hautveränderungen auch schon klinisch ganz und gar nicht principiell rerschieden sind. Denn in gewissen Krankheitsfällen kann man beobachten, dass die beiden Processe direct in einander übergehen, derart, dass zu der Entzündung ein acutes Oedem tritt und umgekehrt ein acutes Oedem eine Entzündung einleitet. Dieses sind so bekannte Vorgänge, dass man schon das Bedürfniss gefühlt hat, Namen dafür aufzustellen und man bezeichnet daher eine Läsion, bei welcher die erste Art des Ueberganges stattfindet, mit dem Beiwort „urticatus", wie z. B. Roseola urticata, während man bei der zweiten Art von Urticaria „perstans" spricht.

Die nahe Beziehung, welche zwischen Urticaria und den Erythemen schon längst erkannt worden ist, hat also auch für uns ihre Giltigkeit, die wir gefunden haben, dass der den letzteren zu Grunde liegende Process ein ganz anderer ist, als wofür er bisher gehalten worden ist, weil wir eben auch der Urticaria eine andere Pathogenese zuschreiben müssen, die mit der für die Erytheme giltigen so gut wie übereinstimmt.

Das flüchtige Reizödem (Urticaria) ist demnach ein Vorgang, welcher der Entzündung nahe steht und welcher ebenso wie diese durch directe äussere Einwirkung auf die Haut oder auf embolischem Wege hervorgerufen werden kann.

\section{Sch I us s.}

Die in dieser Arbeit aufgestellten Sätze haben, da sie sich mit Thatsachen beschäftigen, für die bisher nur klinische Bezeichnungen in Gebrauch waren, immer noch eine dermatologische Form. In Wirklichkeit aber haben sie einen rein patho- 
logisch-anatomischen Sinn, wie sich schon aus der Darstellung deutlich ergibt, in der wir uns bereits bestrebt haben, an die Stelle der üblichen Namen anatomische zu setzen.

Wir lsönnen daher jetzt anstatt Erythem, in der Bedeutung von Efflorescenz, hämatogene Entzündung und im Sinne ron Dermatose, Blutgefässmetastase sagen und statt von Urticaria, von flüchtigem Reizödem sprechen.

Die allgemein pathologis che Beschreibung der hier behandelten Vorgänge würde dann etwa folgendermassen lauten:

In die Haut gelangen sehr hänfig auf dem Blutwege entzündungserregende Stoffe.

Die durch sie hervorgerufene Entzündung unterscheidet sich klinisch derartig von der gewöhnlichen Form, unter der man sie in der Haut kennt, dass man sie füglich als hämatogene Entzündung ron dieser als exogener trennen kann.

Die hämatogene Entzündung zeichnet sich durch ihren milden Verlauf aus, da ihre Symptome nur aus Hyperämie und Oedem bestehen und alle sonstigen sogenannten Entzündungserscheinungen fehlen. Insbesondere fällt sie auch dadurch auf, dass sie abläuft, ohne Gewebszerstörungen im Gefolge zu haben.

Die bei ihr auftretende Hyperämie hält sich immer in mässigen Grenzen, während das sie begleitende Oedem ein verhältnissmässig starkes ist.

Auf der Haut kommt noch eine schwächere Form der Gefässreizung vor, als die eben besprochene, bei welcher nämlich die Congestion und das sich daran anschliessende Oedem einen ausserordentlich schnellen Ablauf nehmen, der nur nach Minuten oder Stunden zählt. Histologisch fehlt hier Emigration und der Fibringehalt des Transsudates.

Die hämatogene Entzündung kann, ihrer Entstehung gemäss, auf einmal diffus ganze Regionen befallen, oder auch in isolirten kleinen Herden auftreten.

In letzterem Falle beobachtet man in kurzer Zeit die Entwicklung zahlreicher isolirter Entzündungsherde, die über die ganze Körperoberfläche symmetrisch vertheilt sind.

Besonders häufig localisiren sich dieselben an den Extremitäten und an dem Kopfe und in der ersteren Region sind die Streckseiten berorzugt. 
Man kann demnach auf der Hautoberfläche geradezu Praedilectionsgebiete für Blutgefässmetastasen unterscheiden.

Die hämatogene Entzündung kommt in der Haut auch als Einleitung von anderen pathologischen Veränderungen vor, die wie Eiterung, Nekrose, Granulombildung sich je nach der betreffenden Ursache, die auf embolischem Wege in die Haut gelangt ist, entwickeln.

Bei der hämatogenen Entzündung gibt es Gefässveränderungen, die nur mikroskopisch zu erkennen sind, wie Stasis, Thrombose, Wandentzündungen.

Mit besonderer Häufigkeit sind in der Haut diese Gefässalterationen nicht an dem arteriellen, sondern an dem venösen Abschnitte localisirt.

Derartige primäre Venenentzündungen, acuter und chronischer Natur, nun haben ihren Sitz häufig in den tieferen Cutisschichten und in der Subcutis und sind dann klinisch als tiefsitzende Knoten, in welcher Form sie gleich von Anfang an auftreten oder zu der sie sich allmälig ausbilden, erkennbar.

Die Erklärung der Abbildungen auf Taf. I.-VI. ist dem Texte zu entnehmen. 


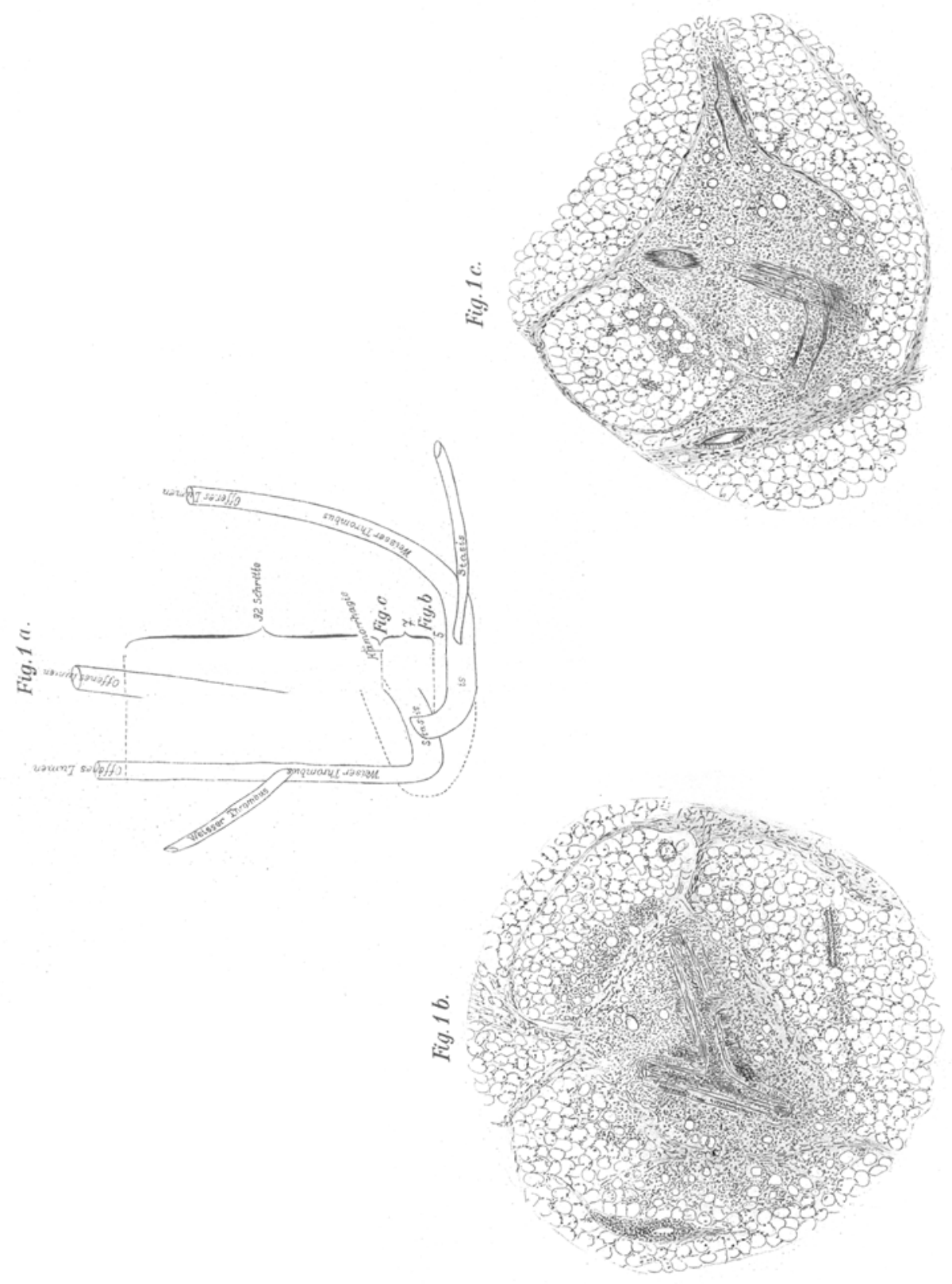




\section{Fig. 2}

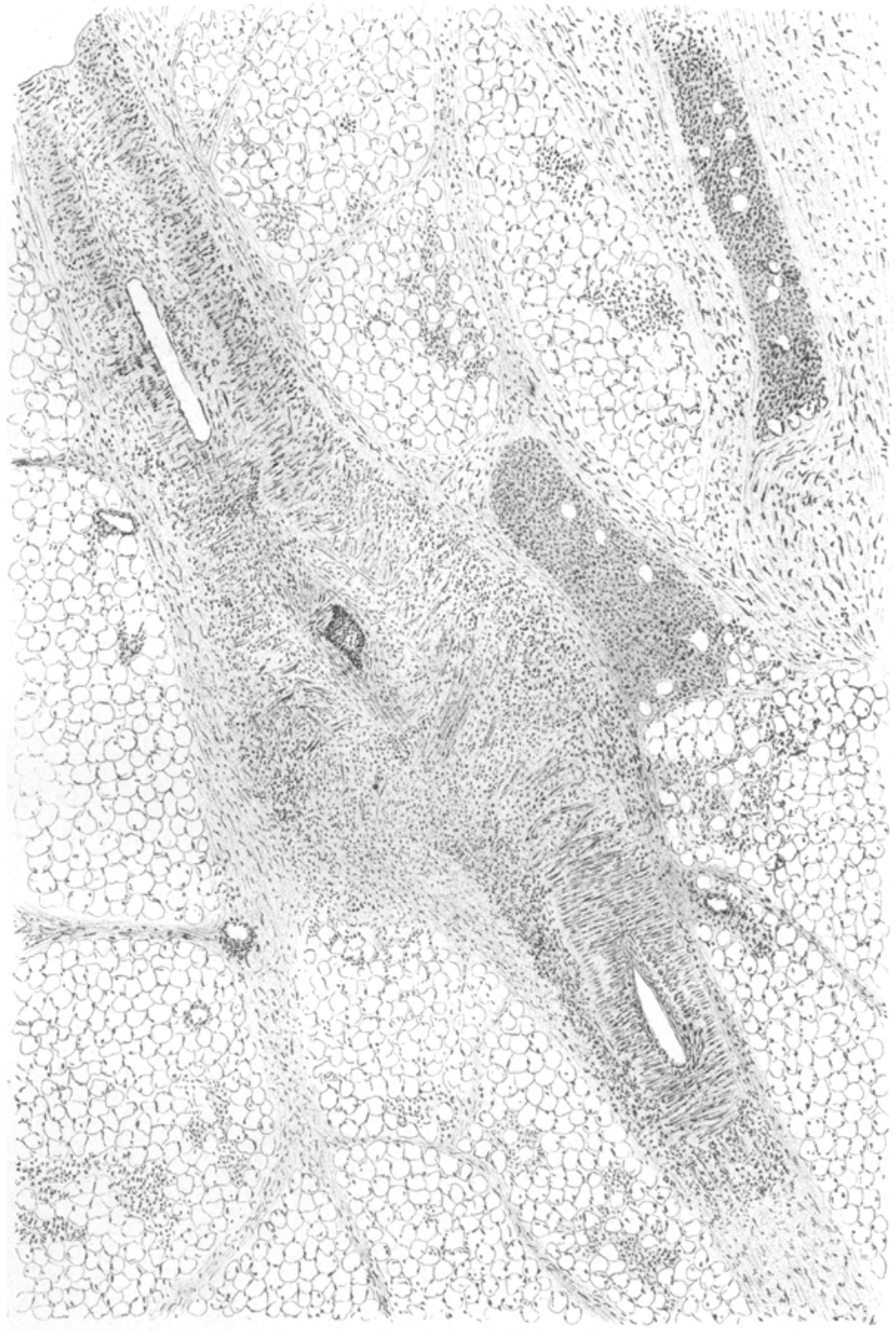

Philippson: Über Embolie und Metastase in der Haut. 


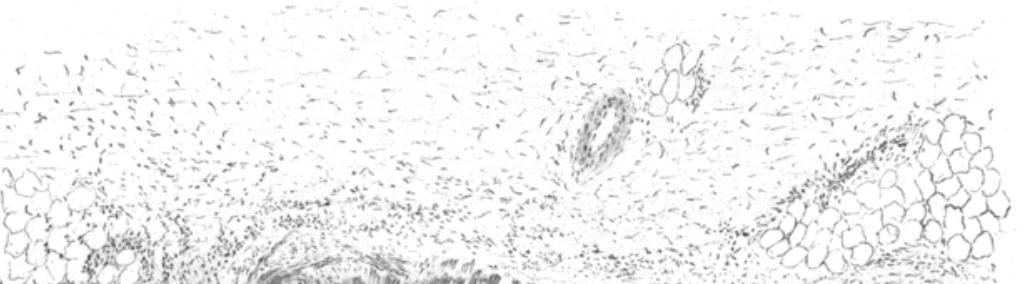

Fig 3.

in 3 (3)

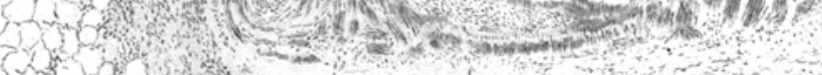
1

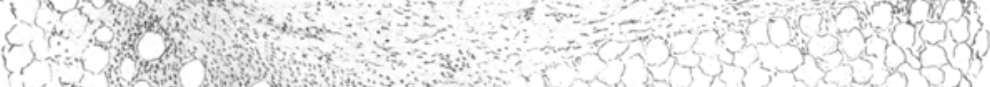
$\{3$ (4)

Fig.'́ a.
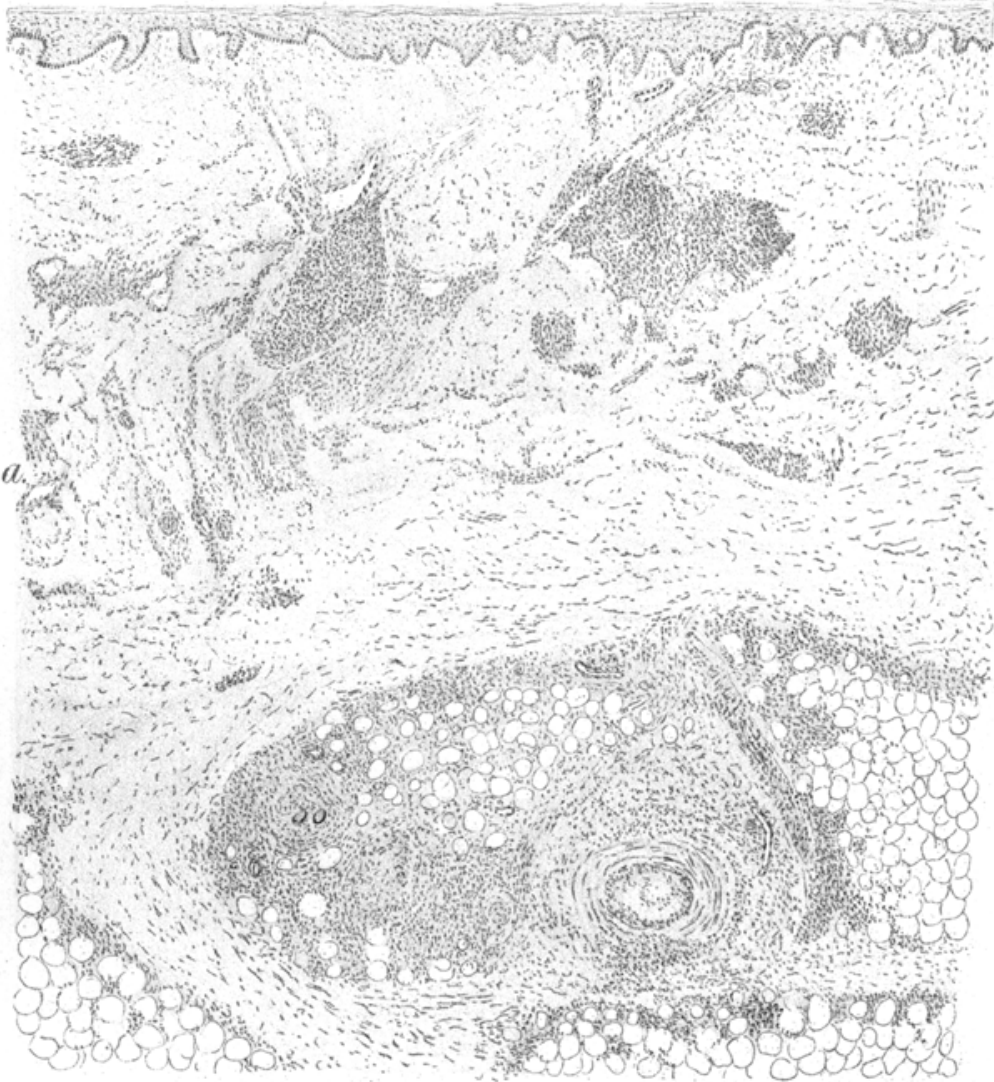

Philippson: Über Embolie und Metastase in der Haut. 


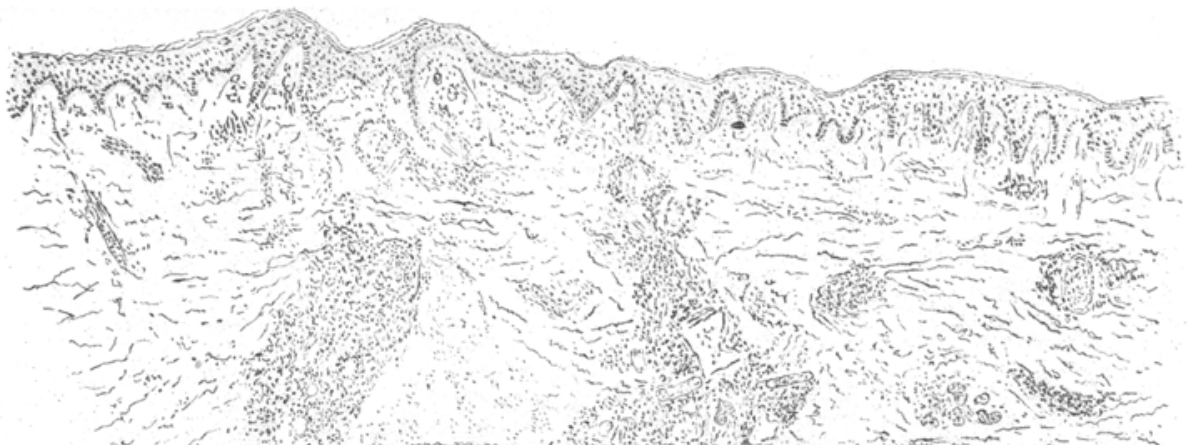

Fig. $4 b$.
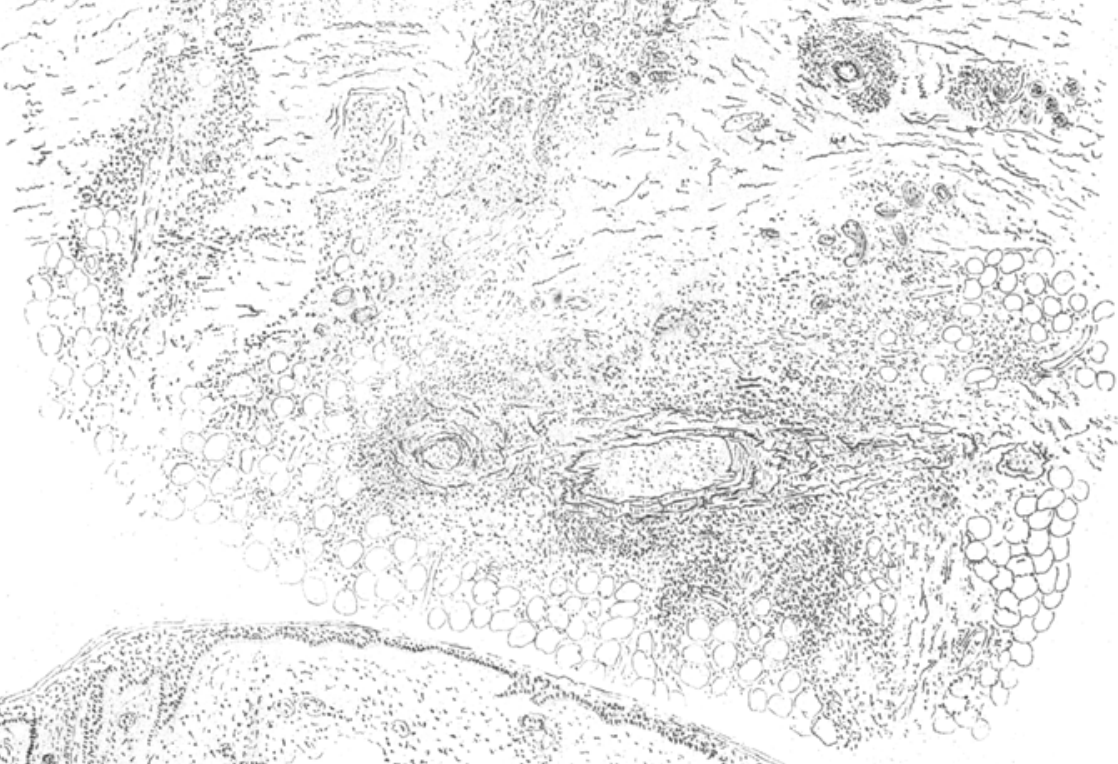

Fig. $4 c$.

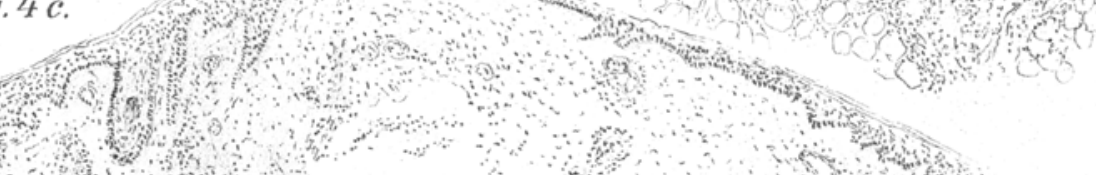

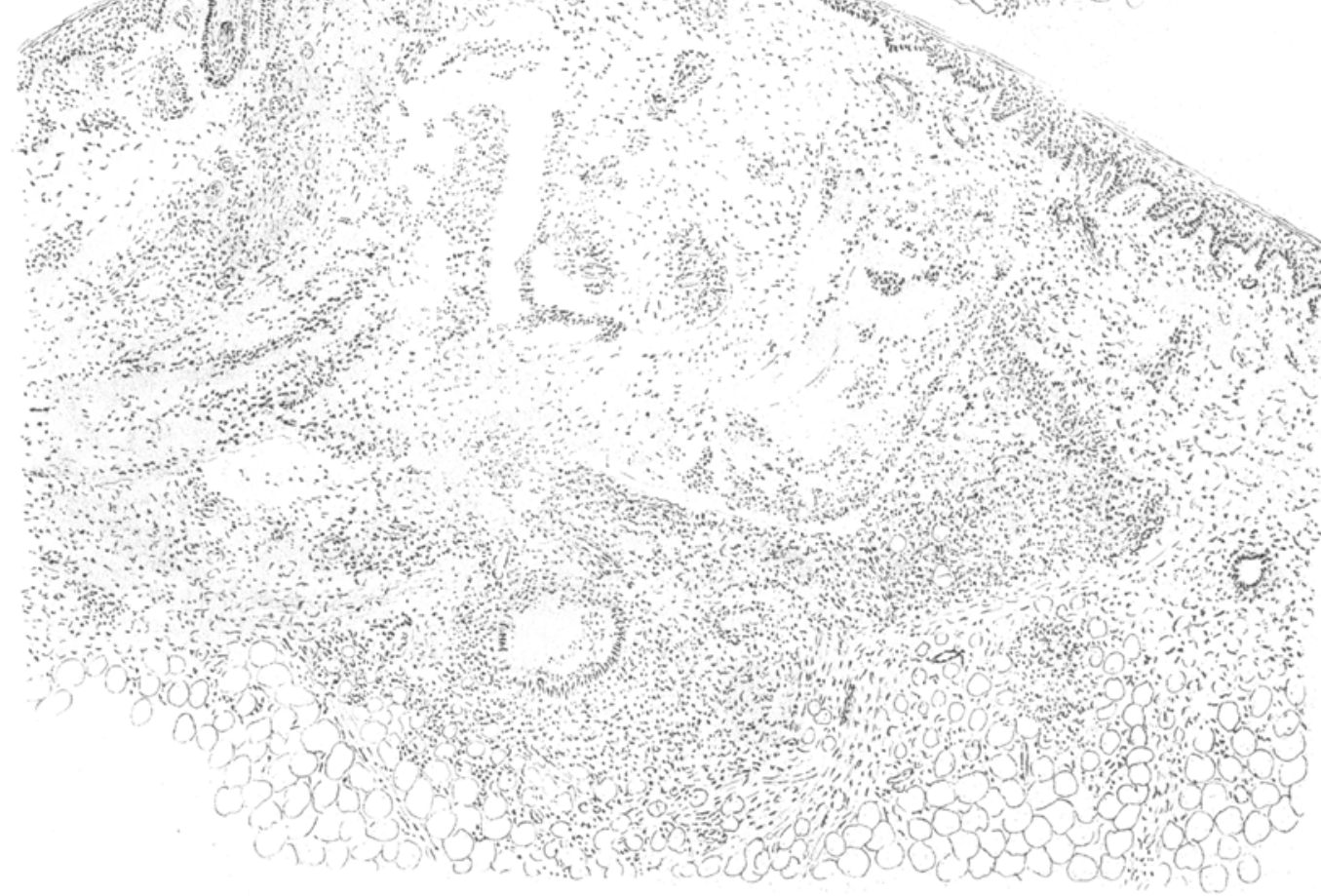

Philippson: Über Embolie und Metastase in der Haut. 

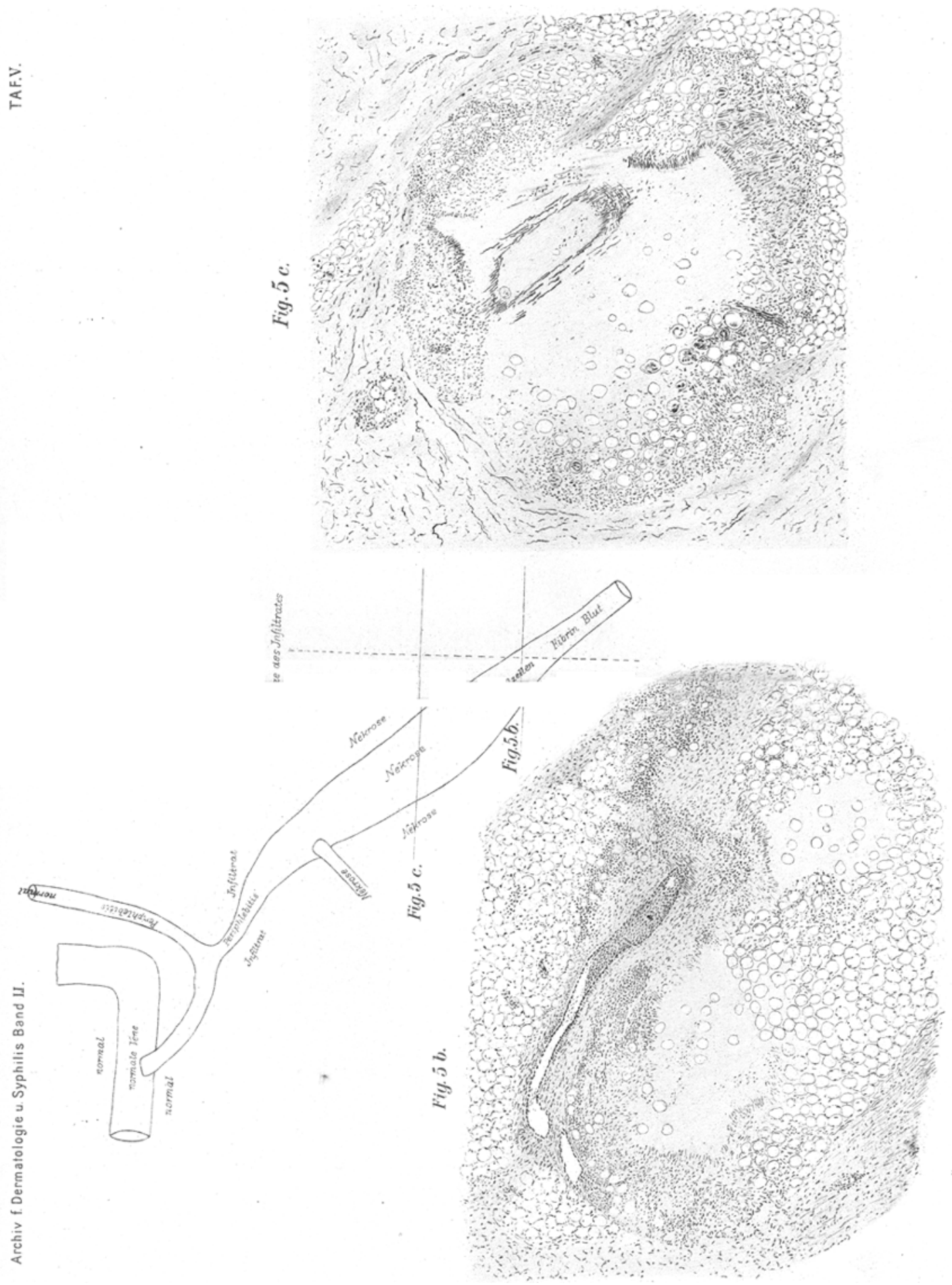

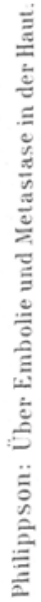



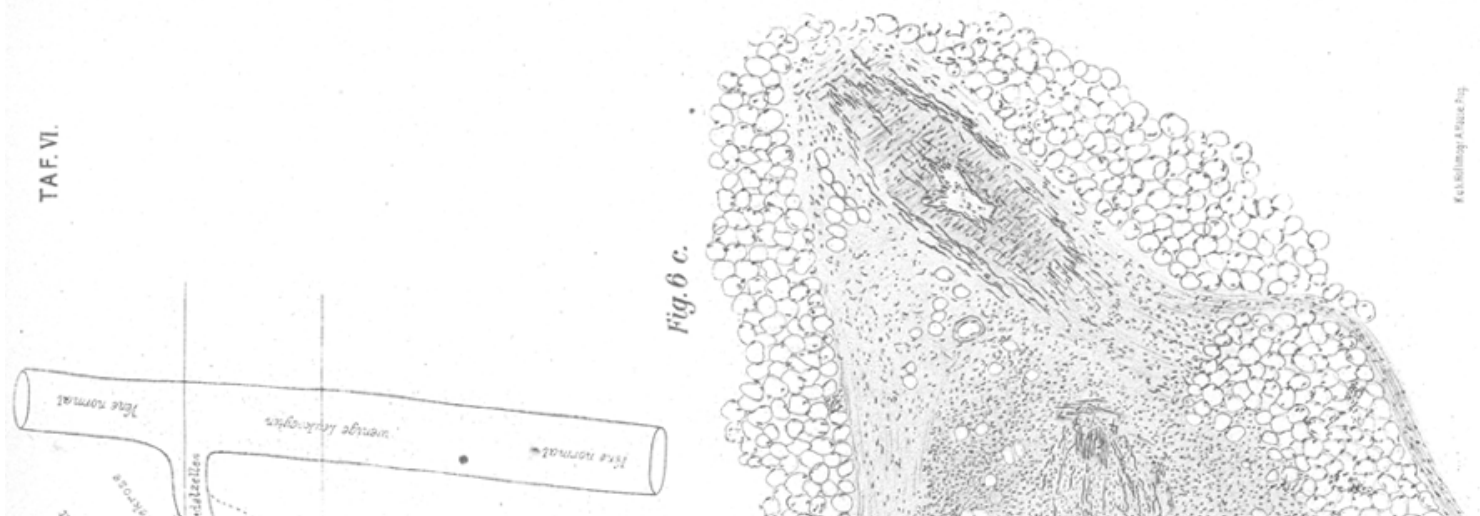

¿ 